\title{
2 Wissenschaft als Literatur: Poetik und Hermeneutik der literarischen Transformation wissenschaftlicher Diskurse
}

Was geschieht, wenn wissenschaftliche Methoden, Theorien, Wissensmodelle und Repräsentationsformen literarisch-produktiv rezipiert werden, und was geschieht, wenn wir als Leser ,Wissenschaftsliteratur' rezipieren? Mit dieser gezielt schlicht formulierten Frage ist zwar eine unilaterale Transferrichtung (aus Wissenschaft in Literatur) angezeigt; diese schließt jedoch, wie bereits einleitend signalisiert, die gründliche Rezeption jener wissenschaftlichen und wissenschaftsgeschichtlichen Diskurse, die für einen literarischen Text produktiv gemacht wurden, mitnichten aus. Vielmehr wird der hier gesetzte Primat der Ästhetik und der Fiktion gerade auch im Studium der wissenschaftlichen Diskurse die Produktivität der Möglichkeiten des Freilegens, Produktivmachens oder Auserzählens literarischer Funktionen erweisen. Dabei gilt es, die Verhältnismodalitäten von Wissenschaft und Literatur „zu beobachten[,] sie zugleich als Produkt einer Beobachtung zu begreifen“248 und entsprechend $\mathrm{zu}$ beschreiben. In produktionsästhetischer Perspektive werden - v.a. im Rückgriff auf Isers Grenzüberschreitungstheorie sowie intertextualitätstheoretische Überlegungen - zunächst Komponenten und Strategien des Transfers näher in den Blick genommen, um sodann in rezeptionsästhetischer Perspektive die Bedingungen der Möglichkeit der Realisierung dieses ,Dialogs der Texte‘ zu hinterfragen.

248 Nicolas Pethes: Poetik/Wissen. Konzeption eines problematischen Transfers, in: Romantische Wissenspoetik. Die Künste und die Wissenschaften um 1800, hrsg. v. Gabriele Brandstetter u. Gerhard Neumann, Würzburg 2004, S. 341-372, hier S. 368. Dass damit nicht - wie bei Pethes - zwangsläufig eine system- oder diskursanalytische Fundierung des Vorgehens einhergeht, räumt der Verf. selbst ein: „Eine ,Wissenspoetik‘, die die Frage nach dem Transfer mit Foucault und Luhmann stellen möchte, wird weder von hermetischer Systemgeschlossenheit noch von einem transdisziplinären Einheitsdiskurs ausgehen, sondern zu beschreiben versuchen, wie kommunikative Ereignisse zwischen den Diskursen/Systemen kursieren“ (ebd., S. 369). 


\subsection{Zum Transfer naturwissenschaftlicher Diskurse ins Medium der Literatur}

\subsubsection{Komponenten des Transfers: Wiederholung und Transgression}

Indem wissenschaftlich gesichertes Wissen durch den Transfer in Literatur in eine Welt des Als-ob und damit unter den Primat der Fiktion gestellt wird, wächst ihm eine andere Seins-, Wahrheits-, Erkenntnis- und Funktionsweise zu: Die streng determinierte, eindeutig-propositionale Aussage des wissenschaftlichen Urteils verwandelt sich in die offene, undeterminierte, nichtpropositionale Behauptung der Dichtung und somit in ein Konstituens der darin entworfenen fiktionalen Welt. Die poetisierte wissenschaftliche Aussage bleibt zwar verwiesen auf den epistemologischen Zusammenhang und damit auf jenen wirklichen Sachverhalt, der mit dem epistemischen Urteil identifikatorisch ausgesagt wird, ist mit diesem jedoch nicht länger zu identifizieren. Im Transfer wird die epistemische Aussage (und d.h. immer auch die mit dieser zur Aussage gebrachte Wirklichkeit) folglich wiederholt und überschritten zugleich. Wiederholung und Transgression - als die beiden Komponenten des Transfers - vollziehen sich im Akt des Transfers zwar zugleich, müssen jedoch aus heuristischen Gründen getrennt betrachtet werden. Während die Wiederholung den Bezug zur wissenschaftlichen Aussage gewährleistet, folglich den Zusammenhang von Fiktion und Episteme erzeugt und ihr damit eine repräsentierende, konservierende und kontinuitätsstiftende Funktion zukommt, sprengt umgekehrt die Überschreitung diesen Zusammenhang gerade auf und generiert, etabliert und akzentuiert auf diese Weise den Bruch, die Diskontinuität, die Differenz. Bleibt die Wiederholung auf die Regeln des wissenschaftlichen Diskurses, seine Intentionalität und seinen Wahrheitsanspruch verpflichtet (und all dies findet mit der Wiederholung auch Eingang in den fiktionalen Diskurs), so können Diskursregeln, Intentionalität, Wahrheitsanspruch im Akt der Transgression außer Kraft gesetzt oder neuen Diskursregeln, Intentionen, Erkenntnismöglichkeiten, pragmatischen Zwecken usw. ,unterworfen` bzw. verfügbar gemacht werden. ${ }^{249}$

249 Die hier vorgenommene Beschreibung der Transferkomponenten und - wie im Anschluss $\mathrm{zu}$ zeigen sein wird - der damit verbundenen konkreten produktionsäthetischen Strategien deckt sich im Resultat weitgehend mit Klinkerts systemtheoretischer Begründung des Verhältnisses von Literatur und Wissenschaft: „Letztlich haben wir es mit einer doppelten Codierung zu tun: Literatur als Kunst gestaltet sich nach den ästhetischen Prinzipien des Kunstsystems und operiert hierbei autopoietisch; Literatur als Erkenntnismedium setzt sich mit rivalisierenden Erkenntnismedien auseinander und definiert sich in Abgrenzung von diesen“ (Thomas 
Wiederholung und Transgression können im produktionsästhetischen Transfer zwar eine je unterschiedliche Gewichtung erfahren, das für die Gattung poetica scientiae schlechterdings konstitutive Interdependenz- und Interaktionsverhältnis zwischen beiden Komponenten bleibt davon jedoch unberührt. Beide sind stets regulativ und korrektiv aufeinander bezogen, denn zielt die Transgression auf die Akzentuierung der Verschiedenheit von scientia und poetica, mathesis und poesis, von wissenschaftlicher und fiktionaler Behauptung, so steuert die Wiederholung der völligen Auflösung und Trennung dieses $\mathrm{Zu}$ sammenhangs entgegen. Die von beiden jeweils übernommenen Funktionen werden, je nachdem, ob im Transfer das Prinzip der Wiederholung oder das der Transgression dominiert, im fiktionalen Text entsprechend latent oder manifest zur Geltung kommen. Entscheidend ist jedoch, dass die Funktionen der einen durch die Funktionen der anderen zwar verschoben, verdrängt, ausgegrenzt, aber nicht gelöscht werden können. Vielmehr bedingt das Zugleich von Wiederholung und Transgression im Akt des Transfers auch die Interferenz und Interrelation ihrer jeweiligen Funktionen im literarischen Text.

Vor dem Hintergrund der vorgenommenen Differenzierungen kann nun auch das Interdependenz- und Interaktionsverhältnis zwischen Wiederholung und Transgression näher beschrieben werden. Behauptet sich in der Wiederholung die Wirklichkeitsreferenz - im vorliegenden Kontext: die Identität des wissenschaftlichen Diskurses -, wird dieses Bestreben in der Transgression gerade unterlaufen. ${ }^{250}$ Denn die Transgression meint zunächst ja nichts anderes als die Überschreitung des Wiederholten und ist damit kontraproduktiv auf die Wiederholung selbst bezogen. Die Transgression verhindert folglich, dass das Wiederholte identisch ist mit dem, was in der Wiederholung (identisch) wiederholt werden soll. Bezogen auf die Wiederholung (und den in ihr wiederholten realen oder szientifischen Sachverhalt) stellt die Überschreitung stets eine Intervention in Richtung Verfremdung, Überformung oder Negation dar; positiv formuliert und im Hinblick auf die fiktionale Welt wird das Wiederholte derge-

Klinkert: Epistemologische Fiktionen. Zur Interferenz von Literatur und Wissenschaft seit der Aufklärung, Berlin, New York 2010, S. 21). Literatur ist eben „keine Wissenschaft oder Philosophie, sondern ein Medium der Erkenntnis von Erkenntnis“ (ebd.).

250 Auch Gabriel konstatiert für bestimmte Gattungen wie etwa den realistischen oder historischen Roman die Unverzichtbarkeit von „Referenzialisierbarkeit und Verifizierbarkeit“, zugleich aber auch, dass die poetische Darstellung ,dieses Wissen gerade überbietet, indem sie zum Beispiel den Faktenwahrheiten eine symbolische Bedeutung [...] verleiht“ (Gottfried Gabriel: (Gottfried Gabriel: Fiktion, Wahrheit und Erkenntnis in der Literatur, in: Wahrheit, Wissen und Erkenntnis in der Literatur. Philosophische Beiträge, hrsg. v. Christoph Demmerling u. Ingrid Vendrell Ferran, Berlin 2014, S. 163-180, hier S. 170 f.). 
stalt freigesetzt, dass es auch als Zeichen für etwas anderes verfügbar gemacht wird. Analoges gilt in der Umkehrung: Indem die Wiederholung in erster Linie darauf zielt, einen (wissenschaftlichen) Sachverhalt und den in ihm angelegten Informationswert identisch wiederzugeben, steuert auch sie der Transgression und den mit dieser gegebenen ,Gefahren' ihrer Verfremdung etc. entgegen, bringt ihre repräsentative Funktion - in welcher Gestalt auch immer - zur Geltung und bleibt damit als faktisch nicht aufzuhebendes „(wissenschaftliches) Realitätsprädikat“ im literarischen Text präsent. Damit kann die Beziehung zwischen Wiederholung und Transgression als eine dialektische beschrieben werden: Das, was durch das jeweils eine zur Positionierung, Gestaltung oder Organisation gebracht werden soll, geschieht stets durch Negation dessen, was durch das jeweils andere zu seiner Positionierung, Gestaltung oder Organisation drängt. Was der in der Wiederholung auf seine Repräsentation zielende wissenschaftliche Sachverhalt in der Transgression an Reduktion, Substanzverlust, Verfremdung, Irrealisierung, De-Epistemologisierung ,erleidet', wächst der fiktionalen Welt an Substanz, Realität und Gestaltungsmöglichkeiten zu - und umgekehrt. ${ }^{251}$

Die der Gattung eigene Hybridität gründet vor allem darin, dass Wiederholung und Transgression, unabhängig davon, mit welcher Dominanz sie sich in der fiktionalen Welt behaupten, sich gegenseitig nicht auslöschen können, ohne ihren gattungspoetologischen Status einzubüßen. Dieser impliziert gleichsam die äußerste Grenze dessen, bis zu welchem Grad sich Wiederholung und Transgression im produktionsäthetischen Transfer durchsetzen dürfen und auch durchsetzen müssen: So muss das Wiederholte in seiner Eigenschaft als ein bestimmtes Realitätsprädikat (Wissenschaftsprädikat) seine Relevanz zumindest soweit behaupten, dass seine Wiedererkennung und Konkretisation durch den Leser potentiell gewährleistet bleibt; desgleichen muss die Transgression in ihrer Eigenschaft, das Wissenschaftlich-Reale zum Zeichen für etwas anderes freizusetzen und damit die Differenz zwischen Welt der Wissenschaft und Welt der Fiktion zu etablieren, seine Relevanz in dem Maße behaupten, dass das in der Wiederholung Repräsentierte als ein fiktional Überschrittenes (und in dieser Überschreitung immer auch Freigesetztes und Freiverfügbares) entsprechend markiert ist.

Auf die Frage, die sich von hier aus stellt, nämlich welche Bedingungen erfüllt sein müssen, damit ein literarisches Werk der Gattung poetica scientiae zuzurechnen ist, kann lediglich eine vorläufige Antwort versucht werden. Die

251 Wiederholung als conditio sine qua non der Transgression, das Identische als Voraussetzung für die Etablierung von Differenz. 
Quantität der wissenschaftlichen Bezüge und die Qualität ihrer Repräsentation scheinen nur wenig verlässliche Kriterien zu sein. Die Antwort, so steht zu vermuten, ist vor allem in jener durch den Transfer etablierten Differenz von scientia und poetica, ihrer produktiven Funktionalisierung auf den und für die unterschiedlichen Ebenen des Erzählens sowie in der Nachhaltigkeit zu suchen, mit der sie den Lektüreprozess und damit das, was in der Lektüre jeweils zur Konkretisierung gelangt, zu steuern vermag.

\subsubsection{Transferstrategien: Akte des Fingierens und Intertextualität}

Als Ergebnis der Transgression des Wiederholten ist die gattungskonstituierende Differenz von scientia und poetica stets als eine Differenz von Identität (das Wiederholte) und Differenz (das fiktive ,Andere‘ des Wiederholten) zu charakterisieren. Die durch Wiederholung und Transgression etablierte Differenz von Identität und Differenz, somit also das Verhältnis, in das beide Differenzaspekte zueinander gesetzt sind, kann mittels Transferstrategien jeweils unterschiedlich akzentuiert werden. Transferstrategien sind damit Regel- und Steuerungsmechanismen, die das Verhältnis von Wiederholung und Transgression, von Identität und Differenz und damit die je verschiedene ,Phänomenologie‘ und Funktionalität der für die Gattung konstitutiven Differenz von scientia und poetica modellieren und regulieren. Unter der Voraussetzung, dass die Differenz von Wirklichkeitsbezug und Fiktionalität für jede Literatur geltend $\mathrm{zu}$ machen ist, kommt den im Folgenden vorgestellten Transferstrategien keine Exklusivität für die in Rede stehende Gattung zu; ferner entspricht die Vielfalt dieser Strategien der Vielfalt narrativer und fiktionaler Möglichkeiten. Es wird also vor allem darum $\mathrm{zu}$ tun sein, einige wenige dieser für jeden produktionsästhetischen Prozess relevanten Grundoperationen zu erinnern und diese mit Blick auf die der poetica scientiae eigenen Möglichkeiten, Leistungen und Funktionen zu spezifizieren.

\section{Akte des Fingierens: Selektion, Kombination, Entblößung der Fiktionalität} Die basalen Strategien des Transfers, mittels derer die Differenz von scientia und poetica allererst etabliert und akzentuiert wird, können mit Wolfgang Iser als „Akte des Fingierens“ bezeichnet werden. Iser unterscheidet dabei drei Operationen, die für die Konstituierung des Fiktiven ${ }^{252}$ maßgeblich sind und in je-

252 Das Fiktive bestimmt Iser als die „Übersetzung des Imaginären in die konkrete Gestalt zum Zwecke des Gebrauchs“ (Wolfgang Iser: Akte des Fingierens. Oder: Was ist das Fiktive im 
weils „bestimmte, voneinander unterscheidbare Gestalten ausmünden“: 1 . die Selektion von Elementen aus der realen Umwelt; sie erweist sich als „Möglichkeit, die Intentionalität ${ }^{253}$ eines Textes zu fassen, denn sie bewirkt es, dass bestimmte Sinnsysteme der Lebenswelt zu Bezugsfeldern des Textes und diese wiederum zum Kontext wechselseitiger Auslegung werden“;254 2. die Kombination der „aus der Textumwelt selektierten Elemente“ sowie der „Schemata des Textes selbst“; ${ }^{255}$ die dadurch erzeugten innertextuellen Relationierungen ${ }^{256}$

fiktionalen Text?, in: Funktionen des Fiktiven [= Poetik und Hermeneutik X], hrsg. v. Dieter Henrich u. Wolfgang Iser, München 1983, S. 121-152, hier S. 134). Damit ist das Imaginäre keineswegs ein den Formen des Fiktiven vorbehaltenes Vermögen: „Es ist mithin nicht die imaginative Dimension, die das literarische vom außerliterarischen Wissen trennt, sondern deren Formung durch die Fiktion. Die Imagination versieht in sämtlichen Bereichen unserer Wissensordnung die Aufgabe der Vorstellungssimulation durch Bildproduktion [...]. Erst in dem Moment, da diese Bildproduktion vom Akt des Fingierens instrumentalisiert und mit den Funktionsmodellen poetischer Gattungen bzw. Stilmittel verknüpft wird, gewinnt sie eine literarische Qualität“ (Peter-André Alt: Beobachtungen dritter Ordnung. Literaturgeschichte als Funktionsgeschichte kulturellen Wissens, in: Grenzen der Germanistik. Rephilologisierung oder Erweiterung?, hrsg. v. Walter Erhart, Stuttgart, Weimar 2004, S. 186-209, hier S. 191).

253 Die im fiktionalen Text nicht explizit formulierte Intention ist demzufolge „nur über die Manifestationsqualitäten einzukreisen, die sich in der Selektivität des Textes im Blick auf seine Umweltsysteme erkennen lassen“. Sie zeigt sich ,in der Dekomposition derjenigen Systeme, an die der Text angeschlossen ist, um sich in diesem Vorgang von ihnen abzuheben“ (ebd., S. 127). Intentionalität erscheint nach Iser als eine „,Übergangsgestalt‘ zwischen dem Realen und dem Imaginären“; ihr kommt insofern ein Aktualitätsstatus bzw. Ereignischarakter zu, ,als sie sich nicht in der Beziehung der Bezugsfelder erschöpft, sondern diese dekomponiert, um die gewählten Elemente in das Material ihrer Selbstpräsentation zu verwandeln. Aktualität bezeichnet dann das Wirksamwerden des Imaginären im Bereich des Realen“ (ebd., S. 128).

254 Ebd., S. 127.

255 Ebd., S. 129.

256 Wie die Intentionalität als Übergangsgestalt zwischen dem Realen und dem Imaginären erscheint, so ist die innertextuelle Relationierung dadurch, dass sie ihre Faktizität „durch den jeweiligen Grad ihrer Bestimmtheit, aber auch das Einwirken auf jene Elemente, die sie aufeinander bezieht“ (ebd., S. 130), gewinnt, ein Oszillieren zwischen dem Fiktiven und dem Imaginären. Durch Relationierung lassen sich drei Ebenen der Grenzüberschreitung ausmachen: Durch die Relationierung der aus der Textumwelt selektierten Elemente, z.B. der ,in den Text eingekapselten Konventionen, Normen, Werte, Anspielungen und Zitate“, entsteht eine Transgression im Sinne einer „Umgeltung von Geltung“, die durch weitere Relationierungen insofern potenziert werden kann, als ,im literarischen Text bereits durch Relationierung bestimmte semantische Bezugsfelder, die sich ihrerseits über die Relationierung der in den Text eingekapselten Elemente ergeben haben“ (ebd., S. 132), aufeinander bezogen werden können. Dadurch kommt es gleichsam zu einer „Umgeltung jener Geltungen, die sich aus der innertextuellen Organisation semantischer Räume ergeben haben“ (ebd., S. 132). Schließlich erfolgt Relationierung auch auf der „Ebene der lexikalischen Wortbedeutung“, z.B. durch die Neuorganisation 
verwandeln die sprachliche „Funktion des Bezeichnens in eine solche des Figurierens"; damit verliere Sprache zwar ihren denotativen, nicht aber ihren Verweischarakter; ${ }^{257}$ schließlich 3. die Entblößung der Fiktionalität durch sogenannte Fiktionssignale; diese bezeichnen nicht die Fiktion schlechthin, sondern einen auf historischen Konventionen beruhenden und entsprechend variablen „,Kontrakt' zwischen Autor und Leser, dessen Regelungen den Text nicht als Diskurs, sondern als ,inszenierten Diskurs“ ausweisen“; ${ }^{258}$ die Gestalt, in welche die fiktionale Selbstentblößung mündet, ist die „Einklammerung“, durch die „alle Welt, die im literarischen Text organisiert ist, zu einem Als-ob“ wird. ${ }^{259}$

von konventionell gebrauchten Wortfeldern. Auch in diesem Fall werden ,schwebende، Beziehungen erzeugt, die sich durch „Instabilität“ auszeichnen, wodurch ein „semantisches Spektrum“ erzeugt wird, das sich „auf keine der kombinierten Wortfelder mehr zurückbringen lässt“ (ebd., S. 129). - Intentionalität und Relationierung erweisen sich gleichermaßen als „facts from fiction“ (Goodman), deren Eigentümlichkeit darin besteht, „daß sie weder eine Qualität dessen sind, worauf sie sich beziehen, noch [und dies gerade aufgrund ihrer durch diese Bezugnahme gewährleisteten Bestimmtheit] eine solche, die mit dem Imaginären überhaupt identisch wäre. Daher ließe sich das Fiktive als eine eigentümliche Übergangsgestalt qualifizieren, die sich immer zwischen das Reale und das Imaginäre zum Zweck ihrer wechselseitigen Anschließbarkeit schiebt“ (ebd., S. 150). - Damit sind Intentionalität und Relationierung zwar „,̈̈ber die von ihren in der Sprache gezeigten Auswirkungen zugänglich“ (ebd., S. 133), ohne aber selbst Sprache zu sein. Vielmehr besitzen sie den „Status der Aktualität“ und haben damit „Ereignischarakter“ (ebd., S. 128): Dieser wird im Fall der Intentionalität dadurch erzeugt, dass sie „sich nicht in der Bezeichnung der Bezugsfelder erschöpft, sondern diese dekomponiert, um die gewählten Elemente in das Material ihrer Selbstpräsentation zu verwandeln“ und damit ihre Aktualität im „Wirksamwerden des Imaginären im Bereich des Realen“ zu bekunden (ebd., S. 128) und im Fall der Relationierung dadurch, dass durch die Verletzung und Transgression primärer und - da bereits innertextlich perspektiviert und selektiert sekundärer semantischer Systeme und Diskurse eine neue „semantische Topographie“ (ebd., S. 132) entspringt, die dem Imaginären seine konkrete, wenngleich nicht gänzlich in Sprache ein- und aufgehende Gestalt verleiht (vgl. ebd., S.134). Die Fiktionen - „das Fiktive als eine Konkretisation des Imaginären“ - gewinnen folglich durch die Sprache den „Realitätsanschein, der auf der einen Seite durch die konkrete Gestalt entsteht, die sie dem Imaginären verleihen und dessen sie auf der anderen Seite bedürftig sind, um solchen Gestalten ihre Wirksamkeit zu sichern. Indem sie aber ihre Existenz nur in der Sprache haben, bringen sie sich als solche durch die Art zur Geltung, in der sie die literale bzw. lexikalische Wortbedeutung überschreiten, die Funktion des Bezeichnens stillegen und die sprachliche Unübersetzbarkeit dessen signalisieren, worauf sie verweisen“ (ebd., S. 134).

257 Vgl. ebd., S. 133.

258 Vgl. ebd., S. 135.

259 Ebd., S. 139. Durch dieses Als-Ob ,ist immer die Gegenwart eines Ganzheitsaspektes angezeigt“, in welchem sich „die Funktion des Gebrauchs [abschattet], um dessentwillen die Fiktion ins Werk gesetzt worden ist“ (ebd., S. 139): Denn die Fiktion geschieht immer um ihrer pragmatischen Verwendung willen. Folglich ist die im Text dargestellte Realität auch nicht als 
Das gemeinsame Merkmal dieser Akte des Fingierens besteht darin, dass sie „Grenzüberschreitungen“ darstellen:

Akte des Fingierens sind Grenzüberschreitungen, verwischen aber erstens die Grenzen nicht und zweitens ist dieser Vorgang kein solcher des Transzendierens, weil durch die Akte des Fingierens das jeweils Überschrittene nicht überstiegen, sondern von ihnen ständig parat gehalten wird. Daraus ergibt sich die Doppelung oder Doppelheit als basaler Modus des Fiktiven.

Die Akte des Fingierens als Akte der Grenzüberschreitung implizieren damit jene dialektische Interrelation von Wiederholung und Transgression, wie sie für den Transfer kennzeichnend ist. Die aus ihnen sich jeweils ergebende Dopplung ist letztlich nichts anderes als der Effekt der durch sie - konkret: durch die ihnen implizite Dialektik - etablierten Differenz von Identität und Differenz. Anders formuliert: Die den Akten des Fingierens selbst implizite Dialektik zeichnet diese durch eine operationale - nicht zu trennende, sehr wohl aber zu differenzierende - Doppelfunktion aus, deren Effekt die Dopplung und Doppelfunktionalisierung der ihnen unterworfenen Sachverhalte ist. So ergibt sich im Akt der Selektion die Dopplung dadurch, dass einzelne Elemente aus den Umweltsystemen im Text „aktualisiert“ werden, während andere „inaktiv“ bleiben, doch so, dass sich „Gegenwärtiges aus dem Abwesenden visiert und Abwesendes sich in das Gegenwärtige einzeichnen kann“. ${ }^{260}$ Entsprechend wird im Akt der Kombination ,jedes Wort dialogisch, jedes semantische Feld durch ein anderes gedoppelt, so dass in allem Gesagten immer auch ein anderes zur Geltung kommt“, also auch hier „Abwesendes immer durch Anwesendes gedoppelt ist“.

solche gemeint; sie ist Verweis auf etwas, das sie nicht ist, wenngleich dieses durch sie vorstellbar gemacht werden soll. (ebd., S. 139) Damit erfolgt mit der „Fiktion des Als-ob“ eine „doppelte Überschreitung, nämlich einerseits die Überschreitung der wirklichen Welt in den Akten des Fingierens, und andererseits eine Überschreitung der fiktionalen Welt selbst“. Erst durch diese zweite Transgression, in der die Fiktionalität des fiktiven Textes kenntlich gemacht wird, enthüllt dieser seinen pragmatischen, auf das Reale sich rück- und auswirkenden Zweck, in dem das „,Imaginative““ allererst „,seine zureichende Gestalt gewinnt“ (ebd., S. 141). Dies bedeutet, dass die „dargestellte Welt des Textes noch nicht der Zweck des Textes“ sein kann, sondern als „das wohlbestimmte Vergleichsglied die Bedingung dafür bildet, daß die durch die Klammer angezeigte Verweisung auf die Dimension des Gebrauchs vorstellbar zu werden vermag“(ebd., S. 141).

260 Ebd., S. 126. Die Selektion erfüllt zwei maßgebliche Funktionen: Dadurch, dass „die im Text anwesenden Elemente durch abwesende gedoppelt“ (ebd.) sind, wird das Bezugsfeld überhaupt erst zu einem „Gegenstand der Wahrnehmung“, und dadurch, dass „durch die getroffene Auswahl das mit an[gezeigt] wird, was davon ausgeschlossen ist“, erscheint der Gegenstand der Wahrnehmung in einer bestimmten Perspektive. 
Schließlich ergibt sich die Dopplung im Akt der Selbstentblößung der Fiktion dadurch, dass die Virtualisierung und die mit dieser verbundenen Verfahren und Effekte der Dopplung selbst markiert, die Differenz als Differenz von Identität und Differenz ausgewiesen wird. ${ }^{261}$ Die Entblößung der Fiktionalität ist damit ein selbstreferentieller Akt, dem - und darin zeigt sich ein weiteres Mal die den Akten des Fingierens implizite Dialektik - eine gleichermaßen autoreferentielle, die Identität des fiktionalen Diskurses sichernde, wie heteroreferentielle, die Differenz zu den außertextlichen und textimmanenten Systemen markierende Funktion zukommt.

Das Spezifische der Dopplung - die Dopplung als Effekt der den Akten des Fingierens immanenten Dialektik - ist, dass in ihr real Vorgegebenes zwar jeweils überschritten und d.h. umformuliert, virtualisiert, in seinem Realitätsstatus zum Zwecke der Konfiguration des Fiktiven getilgt und deformiert wird, zugleich jedoch als Hintergrund funktionalisiert ist, vor dem die mit der Transgression einhergehenden Prozesse der Umstrukturierung, Umgeltung etc. allererst thematisch werden können. ${ }^{262}$

Appliziert auf den Gattungstyp der poetica scientiae erscheint es sinnvoll, das Reale, auf das darin Bezug genommen wird, zu unterscheiden in ein lebensweltlich Reales einerseits, in ein wissenschaftlich Reales andererseits. Mit dieser Differenzierung sollen Wissenschaft und Lebenswelt keineswegs voneinander getrennt werden, vielmehr soll damit der unterschiedliche Modus, in dem sich ,Lebensweltliches“ und ,Wissenschaftliches‘ als Reales überwiegend präsentiert, angezeigt werden. Idealtypisch (und damit notwendig simplifizierend) zeigt sich Lebenswelt als Praxis, in gemeinsamen kulturellen Erfahrungen und habitualisierten Vorstellungen, Wissenschaft hingegen als Theorie und Text. Während sich die Lebenswelt gegenüber der Wissenschaft zwar einerseits vielgestaltiger, heterogener und diffuser ausnimmt, so ist sie andererseits auf allgemeine teils natürlich bedingte, teils kulturell tradierte und durch Sozialisation angeeignete Schemata, Muster und Strukturen der Wahrnehmung, des Denkens, des Verhaltens, des Handelns und der Erfahrung zurückführbar, wel-

261 Der fingierende Akt der Selbstentblößung der Fiktion ist somit stets metafiktional funktionalisiert: Insofern sich darin die Dopplung als basaler Modus des Fiktiven - und damit auch die Differenz von Identität und Differenz als Bedingung der Doppelheit - selbst anzeigt, entblößt sich Selbstreferentialität selbst als ein Effekt grenzüberschreitender Operationen; indem im Akt der Selbstentblößung die fiktionale Welt als eine Welt des Als-ob ausgewiesen wird Iser vergleicht sie mit einer in Klammern gesetzte Welt -, werden die Klammern selbst in ihrer parenthetischen Funktion bloßgelegt und damit geöffnet für ihr ,Außerhalb‘.

262 Ausführlich dazu vgl. Wolfgang Iser: Die Doppelungsstruktur des literarisch Fiktiven, in: Henrich u. Iser: Funktionen des Fiktiven, S. 497-558. 
che affektive, soziale, kognitive und kommunikative Einstellungen und damit die Teilhabe an ihr überhaupt erst ermöglichen. Diese allgemeinen formalen ,patterns' gelangen zwar in der konkreten Erfahrung zu einer je unterschiedlichen und individuellen Realisierung, doch ist innerhalb des Gesamtbündels möglicher Attribuierungen stets eine gewisse Teilmenge von Merkmalen gegeben, die sich durch eine relativ hohe Konstanz auszeichnet und in der bestimmten Erfahrung von den Mitgliedern einer bestimmten Kultur regelmäßig besetzt wird. Umgekehrt ist es gerade die Menge der konventionell nicht besetzten Merkmale, welche die Verschiedenheit, Individualität und je Andersartigkeit von Erfahrung ermöglicht. Wird Erfahrung als Erfahrung gewusst, so kann sie mit Aristoteles als ein „Wissen des Besonderen“ definiert werden, das in exemplarischer Weise gleichzeitig eine Wahrnehmung des Allgemeinen leistet. Entscheidend ist, dass die dergestalt ,gerasterte ' und ,stereotypisierte' Lebenswelt - trotz der ihr eigenen Individualität und Heterogenität - ein hohes Maß an Verfügbarkeit und Vertrautheit aufweist. Demgegenüber ist die Text-Welt der Wissenschaft per definitionem eine rational, diskursiv, systematisch und abstrakt verfasste, hochgradig spezialisiert, diszipliniert, propositional ,besetzt ${ }^{\star}$, in ihrer Bedeutung definitorisch eindeutig festgelegt und dem entsprechend in ihrem Wirklichkeitsbezug eindeutig determiniert. ${ }^{263}$ Ihre Sätze und Urteile sind theoretisch - auch dann, wenn es sich dabei um Explikationen eines elementaren Erfahrungswissens handelt. Dadurch sperrt sich das ,wissenschaftlich Reale' einer spontanen, quasi-automatischen Aneignung und Aktualisierung und wird verfügbar nur unter der Voraussetzung ihres rationalen Nach- und Mitvollzugs.

Ihre Relevanz gewinnt diese Differenzierung sowohl für die Akte des Fingierens als auch für den Akt der Rezeption (vgl. unten), denn durch den doppelten Bezug auf Reales verdoppelt sich nicht nur die im Fiktiven aufgehobene, in der

263 Die Wissenschaftssprache ist geprägt durch jenen abstrakten Objektivismus, den zuerst der Bachtinkreis, später vor allem Jacques Derrida als Fiktion des vereindeutigenden, sinnidentischen Wortes kritisiert haben. In ihr sind das einzelne Wort, der einzelne Begriff, das einzelne Urteil monologisch und - weil die ,natürliche', historisch gewachsene Dialogizität des Wortes, seine ihm implizite Alterität, seine ,Spur unterdrückt sind - usurpatorisch, adialogisch und anti-dialogisch. Vgl. Michail Bachtin: Die Ästhetik des Wortes, hrsg. v. Rainer Grübel, übers. v. Rainer Grübel u. Sabine Reese, Frankfurt/M. 1979 u. Derrida: Grammatologie. Mit Lachmann kann deshalb die Wissenschaftssprache als „Gedächtnis des Gesetzes“ bezeichnet werden - im Gegensatz zur dialogischen Sprache der Kultur, die sie als „Speicher des ,lebendigen“ Gedächtnisses“ auffasst (Renate Lachmann: Bachtins Dialogizität und die akmeistische Mythopoetik als Paradigma dialogisierter Lyrik, in: Das Gespräch [= Poetik und Hermeneutik XI], hrsg. v. Karlheinz Stierle u. Rainer Warning, München 1984, S. 489-515, hier S. 501). 
Aufhebung gleichwohl bewahrte Spur dieses Realen, sondern es potenzieren und vervielfältigen sich auch die Möglichkeiten der Kombination der aus Lebenswelt und Wissenschaftswelt jeweils selektierten Elemente und ineins damit die Möglichkeiten ihrer Relationierung, ihrer Semantisierung (Signifikation) und ihrer Verschiedenverstehbarkeit.

Mit Blick auf den literarischen Text erweisen sich empirisch, habituell verfasste Lebenswelt und textuell verfasste Wissenschaftswelt gleichermaßen als außertextliche Bezugsfelder. Dabei erfolgt der Zugriff auf die ,gerasterte' und ,vorstrukturierte' Lebens- und Erfahrungswelt - unabhängig davon, ob vom Autor intendiert oder nicht, unabhängig auch von seiner besonderen fiktiven Gestaltung - stets in einer Weise, dass das in ihr angelegte und von ihr nicht zu trennende Allgemein-Exemplarische mittransferiert, mitrepräsentiert und für seine Konkretisation im Akt des Lesens zugerüstet wird. In der Regel genügt bereits ein minimalistischer Index, um die Vorstellbarkeit oder zumindest das intuitive Verstehen eines auf Lebensweltlich-Reales bezogenen Fiktiven im Rezipienten $\mathrm{zu}$ ermöglichen - unabhängig vom Grad der Reflexion und der Bewusstheit, mit dem diese Konkretisation geschieht. Der Zugriff auf die Welt der Wissenschaft - konkret: auf jenen ,Wissenschaft' genannten Text, der Wissen über die Realität kodiert - hingegen setzt, wie bereits angedeutet, zumindest die partielle rationale Durchdringung des wissenschaftlichen Diskurses voraus, denn erst ihr Studium ermöglicht die Teilhabe an ihr, und erst die Teilhabe an ihr ermöglicht ihre Verfügbarkeit und Freisetzung für literarische Zwecke. ${ }^{264}$ Dies gilt analog, und in verstärktem Maße, auch für den Akt der Rezeption: Denn zur Konkretisation und Vorstellbarkeit eines auf WissenschaftlichReales bezogenen Fiktiven, bedarf es eines Lesers, der die szientifischen Indi$\operatorname{ces}^{265}$ nicht nur als solche $\mathrm{zu}$ identifizieren, sondern diese auch in ihren diskursiven Herkunftszusammenhang $\mathrm{zu}$ integrieren vermag. Zugespitzt formuliert: Während die lebensweltlichen Indices aufgrund der vom Leser (in der Erinnerung) ständig parat gehaltenen Lebenswelt ihre Verweisungsfunktion gleichsam instantan anzeigen, wodurch das, worauf verwiesen wird, in der Vorstellung zur Kopräsenz gelangt, sich als Vergleichsglied zur Welt der Fiktion

264 Damit ist der Zusammenhang von literarischen Formen und Lebenswelt bzw. im Falle der poetica scientiae wissenschaftlichem Wissen einerseits unaufkündbar; andererseits bleibt „das Wissen der Literatur vermöge der Operationen der Fiktion von den Zwecksetzungen und damit verbundenen Begriffssystemen der Wissenschaft getrennt“ (Alt: Beobachtungen dritter Ordnung, S. 192).

265 Der Index kann mehr oder weniger explizit sein; entscheidend ist, dass in ihm Wiederholung und Transgression angezeigt werden, d.h. aufgrund der durchlaufenen Akte des Fingierens ist seine Funktion nie eine benennende, sondern immer nur eine verweisende. 
abschattet, die Differenz zwischen Lebens- und Textwelt ihre Produktivität also entfalten kann, gilt es im Falle der scientifizischen Indices, sofern ihre indexikalische Funktion überhaupt wahrgenommen wird, das, worauf sie verweisen, in der Regel allererst im und für das Leserbewusstsein herzustellen. Wenn die Qualität der Dopplung (Dopplung/Differenz als Effekt der Akte des Fingierens) darin zu sehen ist, dass sie sich im dialektischen Modus von Anwesenheit und Abwesenheit ereignet - Iser spricht von der „Gleichzeitigkeit des wechselseitig Sich-Ausschließenden“266 -, dann muss das im Text Abwesende in der Welt des Lesers in ,irgendeiner Form anwesend sein, soll es sich in das im literarischen Text Anwesende einzeichnen können. Folglich wird sich das auf SzientifischReales bezogene Gegenwärtige des literarischen Textes nur dann aus dem Abwesenden visieren lassen, wenn dieses Abwesende als ein Anwesendes im Leserbewusstsein installiert ist. Erweist sich der szientifische Diskurs, auf den ein literarischer Text Bezug nimmt, im Leserbewusstsein als Leerstelle im Sinne des fehlenden Vorwissens, bleiben die Differenz von scientia und poetica und die aus ihr hervorgetriebenen produktiv-dialektischen und -dialogischen Möglichkeiten deaktiviert, die Gattung selbst unrealisiert und damit als Gattung außer Kraft gesetzt.

Aufgrund seiner allgemeinen Verfügbarkeit und Präsenz, so lässt sich zusammenfassend sagen, weist das Lebensweltlich-Reale eine weitaus größere Disposition zu seiner Aneignung und Aktualisierung auf als das SzientifischReale, dem aufgrund seiner rational-theoretischen Komplexität eine hohe Widerständigkeit gegenüber einer spontanen Aneignung und Aktualisierung anhaftet. Seine Verfügbarkeit als Voraussetzung sowohl für seine Fiktionalisierung durch den Produzenten als auch für die Sinngebungakte durch den Rezipienten erfordert Studium und Arbeit, einen Autor im Sinne des poeta doctus, einen Leser im Sinne des lector doctus. ${ }^{267}$ Ehe im Folgenden die spezifische Rolle des Lesers näher in den Blick genommen wird, gilt es eine Transferstrategie vorzustellen, die sich aus der textuellen Verfasstheit des wissenschaftlich Realen ergibt und deshalb für die in Rede stehende Gattung ein unhintergehbares Konstitutionsprinzip darstellt: die Intertextualität.

266 Iser: Die Doppelungsstruktur des literarisch Fiktiven, S. 503.

267 Fiktionalisierung im Sinne der Etablierung der Differenz von scientia und poetica ist nur unter der Voraussetzung möglich, dass diese Differenz vom Autor erlesen und erarbeitet wurde. Unter der gleichen Bedingung steht, wie noch zu zeigen sein wird, der Vorgang der Semantisierung bzw. der Sinngebung seitens des Lesers. Beide, Produzent und Rezipient, müssen zuerst Leser sein, ehe sie zu Dichtern bzw. Deutern werden. 


\section{Intertextualität}

Ist das wissenschaftlich Reale ein für die Gattung poetica scientiae konstitutives außertextliches Bezugsfeld, das seinerseits textuell verfasst ist, so ist mit Intertextualität zunächst der spezifische Modus beschrieben, in dem diese Literatur auf eben dieses Reale Bezug nimmt. Um den Bezug zwischen Texten im Spielraum seiner Möglichkeiten zu erfassen, gilt es nach Stierle zwischen „semiotischer, phänomenologischer, hermeneutischer und pragmatischer Perspektive“ $\mathrm{zu}$ unterscheiden. ${ }^{268}$ Semiotisch ist die Intertextualitätsrelation eine Verweisung - im Falle der poetica scientiae eine szientifische Deixis -, die dadurch charakterisiert ist, dass der sprachlich organisierte Zeichenzusammenhang des Folgetexts „artikuliert, denotativ“, der Zeichenzusammenhang des Prätextes hingegen „unartikuliert, konnotativ“ gegeben ist: „Der denotierte Text ist in der intertextuellen Relation die Basis des konnotierten Texts.“269 Die Verweisung, die sich durch Verfahren der Referenz auf andere Texte herstellt, kann im Hinblick auf die poetica scientiae als Kontiguitätsfigur der Metonymie präzisiert werden: Die Metonymie als zentrales Verfahren dieser Gattung referiert nicht

268 Karlheinz Stierle: Werk und Intertextualität, in: Stierle/Warning: Das Gespräch, S. 139150, hier S. 143.

269 Stierle: Werk und Intertextualität, S. 144. In der Terminologie Isers ist die semiotische Relation (wie auch die weiteren Relationen) Ergebnis der fingierenden Akte der Selektion und Kombination, wobei die Selektion hier auf der intertextuelle Ebene angesiedelt ist, die Kombination hingegen auf der intratextuellen Ebene; die Kombination ist damit eine Wiederholung der Selektion und deren Funktionen lediglich auf einer anderen Ebene. - In der Selektion werden Auf textimmanenter Ebene geschieht nun in der Kombination dasselbe wie auf der Ebene der selektierten Elemente aus dem Bezugstext, d.h. die Kombination ist eine Wiederholung der Selektion und deren Funktionen auf intratextueller Ebene: Werden in der Selektion einzelne Elemente des Bezugstexts ausgewählt, während andere inaktiv bleiben, dies wiederum so, dass sich die in den Folgetext eingekapselten Elemente des Bezugstexts vor dem Hintergrund dessen, was durch sie ausgegrenzt ist, präsentieren (vgl. Iser: Akte des Fingierens, S. 126), so wird in Kombination (die sich von ...bis erstrecken kann) ,der literale Sinn der Sprache [...] genauso weggeblendet wie ihre Funktion des Bezeichnens“. Diese figurativverweisende Sprachverwendung „lässt sich nicht mehr über bestehende Referenzsysteme einlösen, sondern zielt auf Ausdruck und Repräsentation“, was zum einen bedeutet, „daß dasjenige, worauf verwiesen wird, selbst nicht sprachlicher Natur ist und auch nicht als eine objekthafte Gestalt existiert“, zum anderen aber, dass dadurch die „Vorstellbarkeit dessen, worauf sie verweist“ eröffnet wird, so dass sich schließlich die Sprache „zu einem Analogon [depotenziert], [welches] nur noch die Bedingung möglicher Vorstellbarkeit enthält“, aber eben nicht mit dem, was es vorzustellen gilt, identisch ist (ebd., S. 133 f.): „So bringt sich im Verweis der figurativen Sprache eine eigentümliche Doppeldeutigkeit zum Vorschein: sie funktioniert gleichzeitig als Analogon der Vorstellbarkeit und als Zeichen der sprachlichen Unübersetzbarkeit dessen, was sie anzielt“ (ebd., S. 134). 
auf eine spezifische Realität, sondern auf eine Vielzahl von Ensembles szientifischer Zeichen, die Realität und Realitätserfahrung kodiert haben. ${ }^{270}$ Phänomenologisch gestaltet sich die semiotische Relation von Denotation und Konnotation als ein „Verhältnis von Thema und Horizont“. ${ }^{271}$ Soll dieser Horizont nicht nur ein gewusster, sondern vor allem ein erfahrbarer, ästhetisch gegenwärtiger Horizont sein, so muss die Verweisung, die selbst nicht sprachlicher Natur ist, ihrerseits ästhetisch vergegenwärtigt werden: ${ }^{272}$

Die intertextuelle Gegebenheit ist nicht nur die Funktion eines semiotisch abgerufenen Vorwissens, das der Rezipient ins Spiel zu bringen hat. Der Text vielmehr spielt den Bezugstext herein, und zwar in einer Artikuliertheit, Reliefhaftigkeit, die das Ganze des intertextuellen Bezugstexts nicht einfach als Wissen voraussetzt, sondern es im Medium seiner konkreten Aufgerufenheit erscheinen lässt. Die Weise, wie ein Text eines anderen Texts inne ist, bestimmt seine ästhetische Gegenwärtigkeit. ${ }^{273}$

Im ,Wie‘ des Aufgehoben- und Inneseins wird zugleich das hermeneutischpragmatische Verhältnis - die Haltung und Einstellung - eines Textes zu einem anderen fassbar. Unabhängig davon, ob dieses Verhältnis eines der Applikation, der Überbietung, der Aufbietung einer Autorität, der ironischen Distanznahme, der Erweiterung, der Korrektur usw. ist, unabhängig auch davon, um welche Form der Bezogenheit zwischen Texten es sich konkret handelt - ob der fremde Text als Zitat, Allusion, Reminiszenz in den aktuellen Text eingelagert ist, ob der aktuelle Text sich replikativ, kontrafaktisch, parodistisch oder kommentierend, kritisch, interpretierend zum Referenztext verhält: Grundsätzlich geht es immer „um die semantische Explosion, die in der Berührung der Texte geschieht, um die Erzeugung einer ästhetischen und semantischen Differenz““. ${ }^{274}$

270 Vgl. Lachmann: Bachtins Dialogizität, S. 510.

271 Stierle: Werk und Intertextualität, S. 144.

272 Vgl. ebd. Die ästhetische Erfahrung ist eine Differenzerfahrung, hier konkret: die Erfahrung der Öffnung des Werks auf andere Werke (vgl. ebd.). Grundsätzlich handelt es sich hierbei um die Differenz von fremdem und aktuellem Text. Der fremde Text ist als Text abwesend; im aktuellen Text anwesend ist er als Erinnerung an die Lektüre eines Textes, d.h. als angeeigneter, umgesetzter, in Sinn oder Imagination überführter Text (vgl. ebd. S. 146), anwesend aber auch in den eindeutigen intertextuellen Markierungen, in den Referenzsignalen.

273 Ebd., S. 144 f. In rezeptionsästhetischer Perspektive stellt sich in diesem Zusammenhang erneut die Frage nach den Bedingungen der Konkretisation und damit der ästhetischen Erfahrung, konkret: die Frage nach dem Verhältnis von vorauszusetzendem Vorwissen und Konkretisationsmöglichkeit des literarischen Textes, nach Ab- und Anwesenheit.

274 Renate Lachmann: Ebenen des Intertextualitätsbegriffs, in: Stierle/Warning: Das Gespräch, S. 133-138, hier S. 134. 
Texte, literarische wie nichtliterarische, stehen zueinander nicht nur in einem Verhältnis der semiotischen [und phänomenologischen] Differenz, sondern, auf der Grundlage einer semiotischen Differenz, in einem Sachbezug, der als dieser das Verhältnis der Texte zueinander überschreitet und damit auch die intertextuelle zu einer anderen als intertextuellen Relation macht. ${ }^{275}$

In der Bezogenheit auf eine Sache zeigt sich die intertextuelle Relation im Sinne einer semiotisch-phänomenologischen Differenz stets auch als etwas, das den „Texten in der intertextuellen Relation als das gemeinsame Dritte vorausliegt“ und über die „bloße Textgestalt hinausreicht“. ${ }^{276}$ Für die hermeneutischpragmatische Relation bedeutet das, dass sie sich nur unter der Bedingung ,realisiert‘, dass „Intertextualität überschritten wird“, folglich in der Auslegung und Konkretisation der semiotisch-phänomenologischen Differenz der aufeinander bezogenen Texte.

Die deiktische Funktion, also jene Funktion, welche die intertextuelle Beziehung zwischen Phäno- und Referenztext indiziert, wird durch Referenzsignale garantiert. Bei diesen handelt es sich um Markierungen, die, so Renate Lachmann, die Doppelkodierung manifestieren und folglich als Störung der Textisotopie - als Ambivalenz oder Polyvalenz - wahrgenommen werden. Zwei Beziehungen zwischen Phäno- und Referenztext werden durch die Referenzsignale hauptsächlich angezeigt, die mit Lachmann als Kontiguitäts- und Similaritätsbeziehung bezeichnet werden können. Eine Kontiguitätsbeziehung liegt bei der in Rede stehenden Gattung dann vor, wenn ein konstitutives Element eines fremden, wissenschaftlichen Textes (seine thematische, stilistischrhetorische Ebene betreffend) bzw. eines medial vermittelten wissenschaftlichen Diskurses oder Paradigmas im Phänotext wiederholt wird, das den wissenschaftlichen Referenztext bzw. den wissenschaftlichen Diskurs als Ganzen evoziert, oder wenn eine signifikante Textstrategie eines fremden Textes repräsentiert wird, die den Referenztext in seiner Zugehörigkeit zu einer bestimmten wissenschaftlichen Tradition, Konvention oder Ideologie mit spezifischen thematischen oder stilistisch-rhetorischen Mustern aufruft. Eine Similaritätsbeziehung hingegen ist dann gegeben, ,wenn im Phänotext Strukturen als fremdtextlichen Strukturen äquivalente signalisiert sind; diese Relation realisiert sich nicht in zitierten Elementen oder Verfahren, sondern im Aufbau von analogen Strategien, die ihre Entsprechungen in bestimmten Referenztexten haben. Die Analogie kann hierbei eine formale Äquivalenz bei völliger Umkehrung der

275 Stierle: Werk und Intertextualität, S. 145.

276 Hier und im Folgenden ebd., S. 146. 
Funktion oder die funktionale Äquivalenz bei völliger Umbesetzung der Form bedeuten“. 277

Die für den vorliegenden Problemzusammenhang relevantere Kontiguitätsbeziehung erscheint als Ergebnis der fingierenden Akte der Selektion und Kombination einzelner Elemente oder Strategien eines oder mehrerer Referenztexte. Dabei wird der ursprüngliche Referenzrahmen eines Elements, sein Stellenwert in einer Texttotalität aufgegeben und ein Kontakt zu jeweils anderen fremdtextlichen Elementen hergestellt. Auf diese Weise entstehen heterogene Reihen oder Schichten; einem Vorgang der Zerstreuung folgt der einer Zusammensetzung zu einem neuen Textkomplex. ${ }^{278}$ Die Doppelkodierung des referentiellen Zeichens beruht auf dessen Spaltung in einen (aktuell-)textuellen Verweisungsund Funktionszusammenhang einerseits, in einen (genetisch-)kontextuellen andererseits, wobei die in der Rezeption zu leistende Dekodierung des ersten, der Oberflächenisotopie des fiktionalen Textes gleichsam einverleibten Konnexes nur in Abhängigkeit einer Dekodierung des zweiten, die implikative, die Kontiguitäts-Beziehung zwischen den Texten aufbewahrenden Zusammenhangs erfolgen kann. Als gespaltenes Zeichen verweist das Referenzsignal metonymisch auf den Kontext bzw. das Paradigma, dem es entspringt, d.h. als Teil des Herkunftstextes evoziert es das Textganze (pars pro toto) oder einen größeren Text(sinn)abschnitt (pars pro parte); ${ }^{279}$ zugleich erweist es seine über den Kontext hinausreichende semantisch-funktionale Potentialität, indem es in den Verweisungs- und Funktionszusammenhang des neuen Textes eingeht, „sich diesem zugleich unterwirft und entzieht, einen fremden Text in den Blick bringt und doch auch in diesem nicht aufgeht“. ${ }^{280}$ Auf diese Weise gewinnt das Referenzsignal eine eigentümliche Autonomie, die paradoxerweise aus seiner Partizipation an (mindestens) zwei Texten resultiert, wobei die Partizipation am jeweils einen stets eine (partielle) Emanzipation vom Sinn- und Funktionszusammenhang des anderen bedeutet. Mit anderen Worten: Durch die doppelte Partizipation erleidet das Zeichen eine Heterogenisierung, die seine partizipative Beziehung sowohl zum Referenz- als auch zum Phänotext mit-durchdringt und mit-determiniert. Die Identität des Zeichens besteht dann gerade in seiner Nicht-Identität mit dem ihm in den jeweiligen Texten zugewiesenen Sinn- und

277 Lachmann: Ebenen des Intertextualitätsbegriffs, S. 136. Beide Beziehungstypen schließen einander jedoch nicht aus.

278 Vgl. ebd., S. $136 \mathrm{f}$.

279 Als medial vermittelte können diese Texte bereits in habitualisierte Vorstellungen übergegangen sein.

280 Stierle: Werk und Intertextualität, S. 148. 
Funktionszusammenhang. Nicht zuletzt kommt den Referenzsignalen immer auch eine metapoetische, selbstreflexive Bedeutung zu: Als Zeichen, welche die Intertextualitätsrelation signalisieren und den Ort markieren, an dem anwesender und abwesender Text sich überschneiden, bilden sie auf signifikante Weise auch das Verfahren der Intertextualität selbst zeichenhaft ab. Damit identifizieren sie den literarischen Text insgesamt als Metatext: als Text über einen Text, der an diesen rückwirkend ebenso gerichtet ist wie an jenen potentiellen, ungeschriebenen ,Text', der aus den Lektüre- und Verstehensprozessen durch den Leser resultiert.

Stimmt man mit Stierle darin überein, dass die „Autorität“ eines ästhetischen (auch eines intertextuell organisierten) Textes in der „Bestimmtheit seiner Form“ liegt, ${ }^{281}$ so ist umgekehrt die Autorität eines wissenschaftlichen Textes gerade in der Bestimmtheit und Dominanz des Sachbezugs zu sehen. Diese Unterscheidung erlaubt es, den je spezifischen ontologischen Status literarischer und wissenschaftlicher Diskurse anzuzeigen, ohne ihre gemeinsamen Momente, nämlich ihre grundsätzliche sprachliche Verfasstheit einerseits, ${ }^{282}$ ihre Bezogenheit auf eine Sache andererseits, zu liquidieren. Ferner ermöglicht es diese Unterscheidung, die für die poetica scientiae konstitutiven intertextuellen Relationen gattungsspezifisch zu formulieren.

Ein kurzer Vergleich der beiden Diskursgenres: Der literarische Diskurs ist, wie gezeigt wurde, durch die Differenz von Identität und Differenz und die durch diese bedingte Qualität der Doppelheit charakterisiert. Diese mündet nach Iser in die „Gleichzeitigkeit des wechselseitig Sich-Ausschließenden“, 283 wodurch „dynamisierte Oszillationen“ erzeugt werden, ein Zustand, der zur Vielfalt seiner möglichen Aufhebungen im Prozess der Deutung auseinander treibt; ihm entspringe ein ästhetisches Potential, das als Quelle von Sinn sich schlechterdings durch nichts substituieren lässt. Im Unterschied dazu ist diese Qualität der Doppelheit im wissenschaftlichen Diskurs suspendiert. Dort werden Aussagen und Setzungen vorgenommen, die nicht nur einen logischpropositionalen Wahrheitswert beanspruchen, sondern darüber hinaus eine

281 Ebd., S. 146. Ausdrücklich grenzt Stierle die Autorität der Form, welche die „Identität des Werks bestimmt“, von der „Liberalität der Applikationsmöglichkeiten, der Auslegbarkeiten, Fortführbarkeiten und Bezugnahmen“ (ebd.) ab.

282 Die Dominanz des Sachbezugs im wissenschaftlichen Text meint ja gerade nicht, dass in diesem Momente der Rhetorizität, Metaphorik und Narrativität suspendiert wären, sondern dass der Sachbezug über diese Momente dominiert.

283 Iser: Die Doppelungsstruktur des literarisch Fiktiven, S. 502. 
Identifikation der Setzung mit der zu erkennenden Realität nahe legen. ${ }^{284}$ Während sich der literarische Diskurs durch die Selbstentblößung seiner Fiktionalität einer Falsifizierbarkeit entzieht, ist von ihr die wissenschaftliche Setzung gerade dadurch ständig bedroht, dass diese nicht unter dem Vorzeichen des „Als-ob“ geschieht. ${ }^{285}$

Mit der intertextuellen Integration eines wissenschaftlichen Textes in einen literarischen werden Bestimmtheit und Dominanz des Sachbezugs der Bestimmtheit der fiktionalen Form subordiniert, das Primat der Faktizität dem Primat der Fiktion unterstellt (d.h. semiotisch: die Setzung in eine szientifische Deixis transformiert, phänomenologisch das Thema in einen Horizont, hermeneutisch-pragmatisch: die wie auch immer mit Bedeutung angereicherte, intendierte Verweisung, etwa ironisch, applikativ, überbietend etc.). ${ }^{286}$ Diese durch den Transfer, konkret: den fingierenden Akt der Selektion geleistete Subordination - und daran gilt es immer wieder zu erinnern - bedeutet weder die Eliminierung des Sachbezugs noch die Nichtung der ihn bezeichnenden propositionalen Aussage, sondern beider Wiederholung und Überschreitung zugleich. Dadurch „erfährt die Komplexhaftigkeit der Doppelung eine Steigerung, es entsteht die Gleichzeitigkeit verschiedener Diskurse, die ihre jeweiligen Kontexte als ein Spiel wechselseitigen Auf- und Abblendens entfalten“. ${ }^{287}$ Die aus der intertextuellen Bezugnahme resultierende „semantische Instabilität“ wird durch die im fingierenden Akt der Kombination erzeugten intratextuellen Relationierungen in der Weise gesteigert, „daß die gebündelten Diskurse auch im-

284 Vgl. Iser: „In der Setzung ist die Gleichzeitigkeit des wechselseitig Sich-Ausschließenden aufgehoben, und das bedeutet vielfach, daß die Setzung mit der zu erkennenden Realität identifiziert wird“ (ebd., S. 503).

285 Nach Bachtin hat es das naturwissenschaftliche Erkennen mit dem Ding ohne Stimme zu tun; der literarische Diskurs hingegen ist personal in dem Sinne, dass sich das Subjekt im Dialog, in der Sinnkreuzung mit einem anderen trifft. „,Sinn“ ist personalistisch: in ihm liegen immer Frage, Appell und Vorwegnahme der Antwort, in ihm sind immer zwei (als dialogisches Minimum) gegenwärtig. Es ist dies keine psychologische Personalität, sondern eine SinnPersonalität. [...] Personifikation (in der humanwissenschaftlichen Erkenntnis gegen die Verdinglichung gesetzt) ist jedoch keinesfalls Subjektivierung. Das Extrem ist hier nicht ,ich‘, sondern ,ich“ in Wechselbeziehung mit anderen Personen“ (Bachtin: Die Ästhetik des Wortes, S. 352 u. 354). Entsprechend steht der Monologismus (die Einstimmigkeit) des wissenschaftlichen Diskurses der Dialogik (der Mehrstimmigkeit) der fiktionalen Prosa entgegen.

286 Wobei die Fiktionalisierung bei der in Rede stehenden Gattung immer im Zeichen der szientifischen Fokalisierung steht.

287 Iser: Die Doppelungsstruktur des literarisch Fiktiven, S. 501. 
mer Kontext füreinander sind, weshalb sie jeweils in eine fortlaufend umspringende Thema- und Horizont-Beziehung einrücken“. 288

Mit der Intertextualität als einem für die Gattung poetica scientiae wesentlichen Konstitutionsprinzip rückt ein weiteres Moment in den Blick, welches das Intertextualitätsverfahren selbst - und im Zuge dessen die Gattung - charakterisiert, in der Konsequenz jedoch die gattungspoetologische und -hermeneutische Perspektive für eine gattungsethische öffnet: Intertextualität als ,Dialog der Texte‘. ${ }^{289}$ Dass mit der Rede vom ,Dialog der Texte' weniger eine Definition als vielmehr ein Problem benannt ist, wird unmittelbar einsichtig, sobald man andere, längst eingebürgerte Bezeichnungen für intertextuell konzipierte Texte wie etwa „littérature au second degré“ oder „literary recycling“290 heranzieht. Für den vorliegenden Zusammenhang ist nun nicht die mit diesen Termini insinuierte ästhetische Hierarchie relevant - der Prätext als genialisch verfasstes Original, vor dem sich der Folgetext als künstlerisch minderwertiges Abbild ausnimmt -, sondern die Tatsache, dass damit eine ,Schieflage‘ angezeigt ist, die auch für die dialogische Relation der Intertextualität symptomatisch ist. Gerade die Beobachtung, dass durch den Transfer wissenschaftlicher Texte in Literatur der Primat der Faktizität unter den Primat der ästhetischen Form gestellt wird, lässt die folgende von Stierle geäußerte Vermutung zunächst plausibel erscheinen:

Dialogisch in einem genaueren Sinne kann der Bezug zwischen Texten nicht heißen. Jeder Text macht den hereingeholten Text zum Moment seiner eigenen Bewegung. Dialog setzt die Autonomie der Aktanten des Dialogs voraus. Gerade diese aber erscheint in der intertextuellen Relation aufgehoben. ${ }^{291}$

Vor dem Hintergrund der beschriebenen Transferkomponenten und Transferstrategien, die für die Intertextualität als einem speziellen Verfahren des Trans-

288 Ebd.

289 Dialogische Strukturen manifestieren sich zwar auf allen bisher angesprochenen Ebenen des Transfergeschehens, doch eignet sich die Intertextualität aufgrund des ihr per definitionem eigenen Dialogcharakters besonders gut, die einzelnen Facetten dieses Dialogischen zu analysieren.

290 Zur ,Literatur auf zweiter Stufe` vgl. Gérard Genette: Palimpsestes. La littérature au second degré, Paris 1982; dt.: Palimpseste. Die Literatur auf zweiter Stufe, Frankfurt/M. 1993; zum Terminus des ,literary recycling“ vgl. Peter J. Rabinowitz: „What’s Hecuba to Us?“ The Audience's Experience of Literary Borrowing, in: The Reader in the Text: Essays on Audience and Interpretation, hrsg. v. Susan R. Suleiman u. Inge Crossman, Princeton 1980, S. 241-263, hier S. 246.

291 Stierle: Werk und Intertextualität, S. 147. 
fers zum Zwecke der literarischen Textkonstituierung ja ebenfalls bestimmend sind, erschließt sich die dialogische Relation jedoch als eine weitaus differenziertere und komplexere. Zwei Hypothesen lassen sich den folgenden Ausführungen voranstellen: 1. Die den Akten des Fingierens implizite Dialektik gilt uneingeschränkt auch für die dialogische Intertextualitätsrelation. 2. Entsprechend partizipiert diese dem ,Dialog der Texte‘ implizite Dialektik an der Etablierung und Aufrechterhaltung der gattungskonstitutiven Differenz von Identität und Differenz. Wie das hermeneutische Gespräch vollzieht sich der ,Dialog der Texte، im Modus von Frage und Antwort, als ein verstehendes Einrücken in ein Überlieferungsgeschehen, weicht jedoch vom hermeneutischen Gespräch darin ab, dass er gerade nicht auf die, Verschmelzung der Horizonte‘ im Akt des Verstehens zielt, ${ }^{292}$ sondern auf die nachhaltige Akzentuierung der Differenz, des produktiven ,Widerstreits‘. ${ }^{293}$

Wie nun ist die dem ,Dialog der Texte، implizite Dialektik genauer zu beschreiben? Mit den Akten des Fingierens, die sich stets im Zugleich von Wieder-

292 Vgl. Gadamer: Wahrheit und Methode, bes. Zweiter Teil, Kap. II,1. Dieser Unterschied zwischen dem ,Dialog der Texte‘ und dem hermeneutischen Gespräch bleibt meines Erachtens selbst für den Fall zutreffend, dass die Horizontverschmelzung kein konkret einzulösendes Verstehensziel darstellt, sondern lediglich eine regulative Idee, die zwar angenähert, aber niemals eingeholt werden kann. Entscheidend ist, dass die regulative Idee in einem auf Einverständnis zielenden Verstehensprozess wirksam bleibt, indem sie diesem eine Richtung und einen Richtungssinn vorgibt, ihn also zwar nicht durch eine schon vorentschiedene Antwort, so doch durch eine latent vorgegebene Wahrheit (die formale Wahrheit der Übereinstimmung und des Einverständnisses der Dialogpartner) in seinen Möglichkeiten und seinen Akzeptanzbedingungen begrenzt. Der Dialog der Texte hingegen wird diese regulative Idee - auch in ihrer latenten Wirksamkeit - stets zu negieren suchen. Selbst dann, wenn der literarische Text den Dialog mit anderen Texten pragmatisch aufzuheben versucht - z.B. durch eindeutige Sinnzuschreibungen, utopische Besetzungen etc. - wird die Stimme des fremden Textes stets ein Gegengewicht, einen Widerstand darstellen, der die ,restlose ' Schließung der Differenz bzw. ihre sinnidentische Besetzung verhindert.

293 Die Differenz als das Ästhetikspezifische literarischer Texte berührt sich mit einer postmodern verfassten Hermeneutik der Dispersion, des Dissens, des Widerstreits, wie sie programmatisch von Lyotard beschrieben worden ist (vgl. Francois Lyotard: Der Widerstreit, München 1987). - Nach Iser ist die Differenz von Zeigen und Verschweigen die produktive Bedingung des Dialogs überhaupt. Während diese Differenz in der auf Zwecke gerichteten Alltagskommunikation in der „pragmatischen Finalität des Dialogs“ verschwindet (wobei durch die Aufhebung der Differenz die Zwecke nicht nur ihre Gestalt, sondern auch ihren pragmatischen Charakter allererst gewinnen), kann die Differenz nachhaltig akzentuiert werden, ,indem man das Ungesagte aus der bloß unterstützenden Funktion entlässt und es zur Gleichrangigkeit mit dem Gesagten erhebt; geschieht das, dann wird die offen gehaltene Differenz in eine Zersetzung der Dialogpragmatik umschlagen“ (Wolfgang Iser: Zur Phänomenologie der Dialogregel, in: Stierle/Warning: Das Gespräch, S. 183-189, hier S. 184). 
holung und Transgression ereignen, werden operationale Eingriffe ${ }^{294}$ in einen fremden Text vorgenommen, die dessen Autorität und Identität zum Zwecke der Etablierung der eigenen fiktionalen Autorität und Identität zwangsläufig verletzen: Fiktion, so Iser treffend, ist die „Verletzung der Referenz schlechthin““. ${ }^{295}$ So ist bereits die Selektion ein äußerst distinguierter Vorgang, insofern mit Auswahl und Ausschluss einzelner Textelemente nicht nur Prozesse der Dezentrierung, Reduktion, Fragmentierung, Deformation etc. verbunden sind, sondern auch Entscheidungen, Wertungen und Semantisierungen, die für die Konstitution des Zieltexts ebenso prägend sind wie sie die spezifische Einstellung gegenüber dem Ausgangstext offenlegen. Intertextualität folgt in dieser Betrachtung sicherlich nicht den Regeln des ,herrschaftsfreien Diskurses‘, sondern vollzieht sich - und vor diesem Hintergrund ist Stierles kritische Äußerung zu lesen - als eine textuelle Inkorporations- und Aneignungsbewegung, im Zuge derer das Angeeignete, indem es den Regeln und Zwecken des Zieltextes unterworfen ist, eine Enteignung erfährt. ${ }^{296}$ Den Akten des Fingierens, so ließe sich hermeneutisch formulieren, geht das Gespräch des Autors mit dem Bezugstext und dessen Sinnpositionen voraus, ${ }^{297}$ sie markieren gleichsam das vorläufige Ende dieser kommunikativen Situation, die aber gleichwohl als (Teil-)Antwort (und Antwort meint hier Entschiedenes, Positioniertes, Gesetztes, Bestimmtes, Gewertetes, Gedeutetes) auf jene perspektivierten und interessegesteuerten Fragen, unter denen das Gespräch mit dem Text stattgefunden und auf die der Text seinerseits Antwort - oder eben auch Frage - war, in den zu konstituierenden Text eingeht und das vollendete Werk als (Teil-)Replik auf einen fremden, szientifischen Text ausweist. Jede Wertsetzung fungiert als „Interpretant der Zeichenhandlung“, 298 bedeutet folglich eine zweckhafte Pragmatisierung des

294 Solche Eingriffe sind am Text vorgenommene Handlungen - eben hierin liegt bereits auf dieser formalen Ebene ihre ethische Dimension begründet.

295 Iser: Die Doppelungsstruktur des literarisch Fiktiven, S. 552.

296 Diese ,Enteignung، gilt meines Erachtens nicht nur dann, wenn der aktuelle Text den Referenztext in manifester Weise usurpiert, um ihm seine eigene Autorität aufzuzwingen, sondern auch dann, wenn er den anderen Text affirmiert, seine Sinnintention konserviert usw. Immer wird sich der aktuelle Text durch ein Moment der Differenz vom anderen Text unterscheiden, dessen Bedeutungen, Werte etc. - und sei es noch so subtil - verändern und verschieben.

297 Vgl. Bachtin: Die Ästhetik des Wortes, S. 151 ff.: „Der Autor ist als konstitutives Moment der Form die organisierte, von innen ausgehende Aktivität des ganzen Menschen [...]. In der Einheit des ästhetischen Objektes hat die Beziehung der Form zum Inhalt daher einen spezifischen personalen Charakter, und das ästhetische Objekt ist ein spezifisches, verwirklichtes Ereignis der Aktion und Interaktion von Schöpfer und Werk.“

298 Lachmann: Bachtins Dialogizität, S. 496. 
Dialogs - und damit dessen transitorische Aussetzung, dessen punktuelle Stilllegung im Monologischen. Dass mit der Textkonstitution der Dialog an sein vorläufiges Ende kommt, liegt jedoch nicht nur im replikativen Charakter des Textes begründet, sondern auf einer fundamentaleren Ebene in der Konsolidierung der Zeichenhandlung im Medium der Schrift. ${ }^{299}$

Damit ist jedoch lediglich die eine Seite der Dialektik skizziert: das Moment der Negation und Deprivation, jener dem Dialogischen notwendig implizite Aspekt des Monologischen. Die „Verletzung der Referenz“ ist nicht gleichbedeutend mit Referenzlosigkeit. Die Selektion geschieht, wie alle Akte des Fingierens, als ein Zugleich von Wiederholung und Transgression. Die autoritäts- und identitätsnegierende Aneignungsbewegung vollzieht sich folglich zugleich als eine autoritäts- und identitätsstiftende und -bewahrende Gegenbewegung. Dies gilt für den Ausgangs- ebenso wie für den Folgetext: Der Ausgangstext wird verletzt und zugleich aufgenommen, er wird deformiert, umgedeutet etc. und zugleich in seiner identifizierbaren Andersheit wiederholt und bewahrt; ${ }^{300}$ ebenso sind Identität und Autorität des Folgetextes nicht bloße Effekte der Negation und Deprivation des Ausgangstextes, sondern verdanken sich partiell dessen Identität und Autorität. Anders formuliert: Die Identität des Ausgangstextes scheint als implizite Alterität in der Identität des Folgetextes auf, d.h. Alterität ist ein konstitutives Moment der Identität des Textes selbst und gewinnt nur im Hinblick auf diese ihre spezifische Bedeutung. Damit wird die Differenz in der Replik nicht nur aufgehoben, sondern zugleich entworfen. In diesem „gleichzeitigen Besetzen und Aufreißen der Differenz“ ist nach Iser das eigentliche den Dialog beherrschende Moment zu sehen. ${ }^{301}$ Der Kontiguitätspoetik, die für die Gattung poetica scientiae kennzeichnend ist, entspricht deshalb

299 In Anlehnung an Bachtin lässt sich die Schrift als eine disziplinierende Kraft verstehen, die den Sinnpluralismus und die Vielstimmigkeit einebnet, zugleich aber zum Speicher der Stimmen werden lässt. Durch seine schriftliche Fixierung erleidet die natürliche Dialogizität, das lebendige Gedächtnis des Wortes eine Art vorläufiger Eindeutigkeit; der historisch angereicherte Sinnstrom des Wortes versiegt in der Präsenz des einen Sinns, dessen (auktorial verbürgte) Autorität die „Antwort-Struktur des Wortes, d[ie] Dialogizität als Setzung in der Übersetzung“ (ebd., S. 501) unterdrückt.

300 Diese Aspekte gewinnen dann im Akt der Rezeption noch einmal ein anderes und deutlicheres Gewicht, denn den intertextuellen Momenten eines Textes eignet ein Appellcharakter, die als Anleitung zu einer ,lecture intertextuelle' zu verstehen sind, in deren Folge der Ausgangstext einer Lektüre oder Relektüre unterzogen wird. Diese intertextuelle Lektüre wird stets auch all das am Ausgangstext zutage fördern, was vorher verschüttet lag und erst durch die durch den Folgetext geleisteten Modifikationen, Akzentuierungen etc. zum Vorschein kommen konnte.

301 Iser: Zur Phänomenologie der Dialogregel, S. 185. 
eine Kontiguitätshermeneutik: Ein Verstehen, das sich auf der „Grenze zwischen dem Eigenen und dem Fremden“ ereignet, ${ }^{302}$ sich „eines punktuellen Sinns auf der Grenze zweier Kontexte“"303 bemächtigt und diesen zeichenhaft materialisiert. Dass diese transitorische und punktuelle Wert- und Sinnkonsolodierung nicht in ein den fremden Text dominierendes Sinnmonopol ausufert und damit die Aufhebung des dialogischen Prinzips in einem monologisch erzählenden Diskurs bewirkt, verdankt sich all jenen Formen narrativer Darstellung, die der Inszenierung des Dialogs und der Freilegung des Dialogischen dienen: Strategien der intertextuellen Rhetorik, der chronotopischen Gestaltung, der Differenzierung der Figurenrede und Autorenstimmen, durch die verschiedene Wertakzente, Intentionen, Sinnsetzungen, Weltsichten etc. sich wechselseitig durchdringen, verfremden, kommentieren, hinterfragen, durchkreuzen, bespiegeln und solcherart die Zentrierung, Zementierung und dauerhafte Präsenz eines Sinnes verhindern. In diesem Zusammenhang kommt den Referenzsignalen und ihrer strukturellen Anordnung im Text wiederum eine besondere Funktion zu: Als doppelcodierte Zeichen sind sie nicht nur Statthalter der Alterität, sondern Statthalter der Kontiguität selbst und somit jener Grenze, an denen sich die Texte und mit ihnen der Sinn zugleich berühren und spalten. In dieser grenz-anzeigenden Funktion sind die Referenzsignale - und dies kennzeichnet einen weiteren Aspekt ihrer metatextuellen Qualität - auch Abbildungen der Dialogstruktur selbst. ${ }^{304}$

In eben dieser wechselseitigen und unabdingbaren Bezogenheit, Verwiesenheit und Teilhabe äußert sich das dialogische Moment im Dialektischen, das sich seinerseits als und in der unverzichtbaren Spannung von Deprivation und Freisetzung, Schrift und Stimme, Monologizität und Dialogizität, diskursiver Einheit der Rede und intertextueller ,différance‘, von potentiell unendlicher Verweisstruktur und deren jeweiliger Arretierung zu fiktionalen Prägnanzen ${ }^{305}$ Identität und Alterität, Spaltung und Speicherung, Differenzierung und Spur

302 Bachtin: Die Ästhetik des Wortes, S. 185.

303 Lachmann: Bachtins Dialogizität, S. 495.

304 In diesem Zusammenhang ließe sich von einer artikulierten Intertextualität sprechen: Indem die Referenzsignale die Stimme des fremden Textes markieren, störend in die Isotopie des aktuellen Textes eingreifen und damit die Differenz etablieren, weisen sie das literarische Gespräch nicht nur als ein inszeniertes aus, sondern legen zugleich die Strukturen frei, denen es entspringt. Die Referenzsignale sind Zeichenorte, an denen der fremde Text zur Artikulation kommt, Statthalter seiner Stimme(n), damit auch ganz buchstäblich Referenzerweise an den fremden Text.

305 Renate Lachmann: Zur Semantik metonymischer Intertextualität, in: Stierle/Warning: Das Gespräch, S. 517-523, hier S. 522. 
konzeptualisiert. Hierin wird auch ersichtlich, weshalb der dialogischen Intertextualität (auch in ihrer autoritär-usurpatorischen Form) stets ein literaturkritisches und im weiteren Sinne auch literaturethisches Potential innewohnt: Indem ein literarisches Werk sich als ein intertextuell komponiertes zu erkennen gibt, setzt es sich selbst fragend aus, stellt es sich selbst in seiner Originalität, Abgeschlossenheit und Autorität in Frage.

\subsection{Lector doctus - Die Rolle des Rezipienten}

Seitdem der Leser durch die Rezeptionsästhetik eine entscheidende Aufwertung im literarischen Prozess erfahren hat, ist er aus der literaturwissenschaftlichen Analyse nicht mehr wegzudenken. Erst durch die vom Rezipienten vorgenommenen Konkretisierungen und Aktualisierungen der im literarischen Text angelegten Unbestimmtheits- und Leerstellen, so wissen wir seit den Untersuchungen von Iser und Jauß, entfaltet der literarische Text sein vielschichtiges Potential und wird gleichsam seiner ,Determination', nämlich Literatur zu sein, zugeführt.

Aus der Engführung der Wirklichkeitsreferenz auf szientifische Gegenstän$\mathrm{de}^{306}$ resultiert nun allerdings auch der Entwurf eines Lesers, der aufgrund seines Vorwissens potentiell in der Lage ist, das produktive Potential der für die poetica scientiae schlechterdings konstitutiven Differenz von Wissenschaft und Fiktion zu nutzen. Den beschriebenen poetischen Verfahren und Transferstrategien, mit denen wissenschaftliches Wissen übersetzt, verfremdet und in neue Sinnkreisläufe eingespeist wird, entspricht auf Seiten des Rezipienten eine ebenso komplexe, mit mehrfachen Funktionen besetzte Rezeptionseinstellung. Wenn einerseits der literarische Text nicht die normale Funktion einer wissenschaftlichen Mitteilung haben kann (Dominanz des Poetischen), es primär auch gar nicht darauf ankommt, ob die wissenschaftlichen Bezüge der Regel der Referenz folgen, ob sie also ,wirklich wissenschaftlich stimmig' übernommen wurden, sondern darauf, welche möglichen Funktionen diesem übersetzten

306 Die Referenz auf Wirklichkeit kann eine allgemeine Wirklichkeit meinen, d.h. eine für jedermann greifbare und erfassbare anthropologische Wirklichkeit, ein allgemeiner Erfahrungshorizont. Im Falle der poetica scientiae jedoch wird dieser Referenzbereich enggeführt auf eine szientifische Wirklichkeit und Praxis - oder vielmehr: um diesen Referenzbereich verdoppelt. Dass der szientifische Referenzbereich im fiktionalen Kontext wieder aufgesprengt und erweitert werden kann ins Allgemeine-Wirkliche, Allgemein-Menschliche, ist nur eine von vielen Möglichkeiten der Literatur, beide Referenzbereiche in ein dialogisches Verhältnis zu bringen. 
Wissen im Medium der Literatur zukommt; wenn andererseits in der veränderten Einstellung des Rezipienten gegenüber literarischen Texten die „Richtungsänderung des Bedeutens“, nämlich „weg von der Referenz, hin zum Sinn“307 bereits vollzogen ist, dann stellt sich zunächst die Frage nach dem näheren funktionalen Zusammenhang von Referenz (also direktem Wirklichkeitsbezug) und Sinn. Der Sinn eines literarischen Textes kann zwar auf vielfache Weise realisiert werden und sich in vielen Interpretationen niederschlagen, doch $\mathrm{zu}$ seiner optimalen Realisation wird dem Rezipienten sehr wohl die Fähigkeit abverlangt, den literarischen Text vor der Folie des wissenschaftlichen Textes oder Diskurses zu lesen. Diese doppelte Optik ermöglicht es überhaupt erst, den Wissenstransfer und die damit einhergehenden Prozesse der Bedeutungsänderung und Sinnverschiebung, wie sie sich zwischen wissenschaftlicher Theorie und ästhetischer Praxis ereignen, nachzuvollziehen. Erst durch diese doppelte Optik - zum einen die Rekonstruktion der in die Literatur eingegangenen wissenschaftlichen Diskurseme innerhalb ihres wissenschaftlichen und wissenschaftshistorischen Kontexts, ${ }^{308}$ zum andern durch die Rekonstruktion ihres Funktions- und Bedeutungswandels im literarischen Medium ${ }^{309}$ - wird der literarische Text überhaupt erst als Medium, als eine über sich selbst hinausweisende Vermittlungsinstanz verstehbar. Indem ein literarischer Text identifizierbare und in ihren Kontexten rekonstruierbare Wissensmodelle, Gedankenfiguren, Methoden etc. aufgreift und ästhetisch transformiert, gewinnt er das Potential, auf eben diese Realität wieder zurückzuwirken, im Leser gleichsam eine dritte Optik zu erzeugen, durch die etablierte wissenschaftliche Diskurse neu betrachtet und neu befragt werden können. ${ }^{310}$ Die oben aufgestellte Behauptung, dass sich jeder Wissenstransfer aus der Sicht des jeweiligen Wissenssystems als Reduktion und Verlust darstellt, kann vor diesem Hintergrund dahingehend revidiert werden, dass auch dem Wissenssystem, das durch das Medium der Literatur gleichsam hindurchgegangen ist, etwas zuwächst: neue Wahrnehmungsperspektiven und Fragestellungen, Aufklärung über seinen (intuitiven und spekulativen) Entstehungszusammenhang, über die der Begriffs-, Theorien- und Methodenbildung vorausgehenden und sie begleitenden

307 Gottfried Gabriel: Zwischen Logik und Literatur. Erkenntnisformen von Dichtung, Philosophie und Wissenschaft, Stuttgart 1991, S. 15; vgl. auch S. 154 f.

308 Diese Rekonstruktion kann als der Versuch aufgefasst werden, jene im produktionsästhetischen Transfer geleisteten Wiederholungen zu wiederholen.

309 Dies heißt analog: Rekonstruktion als Wiederholung der produktionsästhetischen Transgression.

310 Erst dieser dritten Optik entspricht auf rezeptionsästhetischer Seite das, was produktionsästhetisch als Transgression bezeichnet wurde. 
narrativen und metaphorischen Prozesse und damit auch über das narrative und metaphorische Element in wissenschaftlichen Schreib- und Darstellungsweisen, eine (Wieder-)Erinnerung daran, dass alles Denken, Erkennen und Phantasieren seinen Ursprung im Menschen nimmt oder ganz einfach ein Bewusstsein von den vielschichtigen Kontexten, in denen ein Wissenssystem steht und aus denen es entsteht. Die Einlösung dieser im weitesten Sinne wissenschaftshermeneutischen Funktionen der poetica scientiae ist dabei dem Leser überantwortet.

Was für die Literatur im Allgemeinen gilt, nämlich dass sie erst durch einen sie aktivierenden und konkretisierenden Leser als Literatur realisiert und ihr ferner möglicherweise eine über sie selbst hinausweisende, nicht nur wirklichkeitsbezogene, sondern auch wirklichkeitsverändernde Relevanz zukommt, gilt für die Wissenschaftsliteratur in besonderem Maße. Denn sowohl zur ,optimalen' Realisierung der fiktionalen Welt als auch im weiteren zur Einlösung darüber hinausgehender, eben z.B. wissenschaftshermeneutischer Funktionen, bedarf es eines dem poeta doctus gleichsam ebenbürtigen Lesers, eines lector doctus, der den angelegten szientifischen Code $\mathrm{zu}$ decodieren und damit jene konstitutive Differenz von scientia und poetica zu aktivieren vermag. ${ }^{311}$ Der Engführung der Wissenschaftsliteratur auf eine primär szientifische Referenz einerseits, der Amplifikation dieser Referenz im Kontext der Fiktion andererseits, korrespondiert gleichsam ein erweitertes Lesermodell: Postuliert wird ein Leser, der sowohl in einen ,lebensweltlichen Erfahrungshorizont' als auch in einen ,szientifischen Wissenshorizont' einzurücken und an beiden zu partizipieren vermag. Zugespitzt formuliert - und hierin unterscheidet sich der in der Wissenschaftsdichtung angelegte und adressierte implizite Leser vom impliziten Leser anderer Gattungen: Der Gattungstyp der poetica scientiae kann überhaupt nur unter der Bedingung ,funktionieren', dass der Leser den an ihn adressierten

311 Vgl. in diesem Zusammenhang auch die Ausführungen von Danneberg und Spoerhase zu ihrer These, dass der ,fiktionale oder faktuale Charakter eines Textes [...] durch den Umgang mit den jeweiligen Texten gestiftet" wird (Lutz Danneberg $u$. Carlos Spoerhase: Wissen in Literatur als Herausforderung einer Pragmatik von Wissenszuschreibungen: sechs Problemfelder, sechs Frage und zwölf Thesen, in: Literatur und Wissen. Theoretisch-methodische Zugänge, hrsg. v. Tilmann Köppe, Berlin, New York 2011, S. 29-75, hier S. 46). Bereits Klinkert formuliert den Befund, „[d]ass Fiktionalität nicht ein inhärentes Textmerkmal ist, sondern ein Operationsmodus, der auf der Ebene des Umgangs mit Texten zur Anwendung kommen kann, aber nicht muss“ (Klinkert: Epistemologische Fiktionen, S. 29). Dem entsprechend sind „,Fiktion“ und ,Wahrheit‘ [...] als kommunikative Operatoren“ zu betrachten, „mit denen Kommunikationsteilnehmer an Texte herangehen und nach denen sie Texte kategorisieren und prozessieren“ (ebd., S. 36). 
szientifischen Code - zumindest partiell - konkretisiert. ${ }^{312}$ Bleibt diese Konkretisation völlig aus, büßt diese Literatur ihren typologischen Status als poetica scientiae ein: sie wird zur ,bloßen' poetica. Zeichnet sich hier einerseits die Gefahr eines elitären Autor-, Literatur- und Leserkonzepts ab, so wird diese Gefahr - und darauf wird im Verlauf dieser Studie immer wieder zurückzukommen sein - durch eine ganze Reihe poetischer Gegenstrategien abgefedert, die gerade auf den umgekehrten Effekt einer De-Elitisierung der Wissenschaft und ihrer ,Personifikationen' zielen.

Trifft die Annahme von dem auch selbstreferentiellen Impetus der poetica scientiae zu, nämlich über die literarische ,Arbeit an der Wissenschaft` die Differenz zur Wissenschaft $\mathrm{zu}$,erschreiben` - ein Impetus, der notwendigerweise auch den (textimmanenten) impliziten Leser ,betrifft‘ - , dann gilt Analoges auch für den empirischen Leser: In rezeptionsästhetischer Perspektive kommt den szientifischen Referenzsignalen eine appellative Funktion zu, die den in der Regel naturwissenschaftlich nur unzureichend gebildeten Leser in ein intertextuell-vergleichendes Lektüreverhalten drängt und ihn unablässig auffordert, im Hin und Her zwischen wissenschaftlichem Prätext und literarischem Folgetext jene für die poetica scientiae konstitutive Differenz auszuloten, ${ }^{313}$ die durch

312 Der Intensitäts- und Genauigkeitsgrad der Konkretisierung kann vielfach variieren, wird jedoch - und dies nicht nur aufgrund der den fiktionalen Texten eigenen Literarizität, sondern auch aufgrund der ,Semiosen' bzw. Weiter- und Umschreibungen, denen nicht-literarische Texte ausgesetzt sind - immer den Status einer lediglich ,synekdochischen“ Konkretisierung haben (Formulierung angelehnt an Wirth, der in anderem Zusammenhang von „synekdochischer Leerstellenergänzung“ spricht, vgl. Uwe Wirth: Die Konjektur als blinder Fleck einer Geschichte bedingten Wissens, in: „Interesse für bedingtes Wissen“. Wechselbeziehungen zwischen den Wissenskulturen, hrsg. v. Caroline Welsh u. Stefan Willer, München 2008, S. 269-294, hier S. 275).

313 Die Differenz ist für den Leser nicht, einfach da', sondern wird durch die intertextuelle Lektüre, die immer auch eine differentielle Lektüre ist, , ausdifferenziert‘ oder - im extremsten Fall - allererst hergestellt. Bei einem (hier freilich nur hypothetisch konstruierten) durchschnittlichen Leser wird es sich in der Regel so verhalten, dass die Referenzsignale die Erinnerung an die Lektüre an den oder die Referenztext(e) auslöst, wobei Lektüre hier auch andere Formen der medialen Vermittlung dieser Referenz-,Texte' einschließt. Der szientifische Sachverhalt, auf den Bezug genommen wird, gewinnt seine Präsenz folglich zunächst im Modus der Erinnerung. Erweist sich diese als lückenhaft und defizitär, wird eben dieses Defizit der Erinnerung als ein Appell zur Relektüre des Prätextes erlebt. Erweist sich die Erinnerung an den Referenztext für das Verständnis des aktuellen Textes als ausreichend, kann von einer ,potenzierten' Rezeptionsintertextualität gesprochen werden, d.h. die Zeichen des Referenztextes sind dem Gedächtnis des Lesers eingeschrieben, die Herstellung dieses Textes obliegt dann der Gedächtnisarbeit des Rezipienten. 
diese Differenz ermöglichten Semantisierungsprozesse einzuleiten und sie für eine ästhetische Erfahrung zu öffnen. ${ }^{314}$

Minimalvoraussetzung dafür, dass diese appellative Funktion überhaupt eine appellative Wirkung im Leser zu erzeugen vermag, ist dessen Kompetenz, die Referenzsignale als solche zu identifizieren und damit die intertextuelle Qualität des Textes und die mit dieser gegebene Ambivalenz oder Polyvalenz die sich zunächst auch als bloße Irritation äußern kann - zu registrieren. Die appellative Funktion, so ließe sich sagen, findet ihre hinreichende Einlösung dann, wenn sie den Leser zu einer Einstellung gegenüber dem Text motiviert, die ganz allgemein als ein ,Verstehen-Wollen' beschrieben werden kann, ${ }^{315}$ das den Eintritt in das dialogische Gespräch mit dem Text stimuliert und initiiert. Den Referenzsignalen kommt dabei eine maßgebliche Funktion zu, insofern sie Explikationen eines dreifach dialogischen Beziehungsgefüges darstellen: Werkästhetisch markieren sie die intertextuelle Relation zwischen Phäno- und Referenztext und die mit dieser gegebenen Doppelzeichenhaftigkeit; darin bezeugen und dokumentieren sie zugleich die vor aller Werkmanifestation vorausgehende Kommunikation zwischen Autor und fremdem Text, verweisen gleichsam selbstreferentiell zurück auf ihren eigenen Ursprung im Gespräch. Diese in den Referenzsignalen manifestierte produktionsästhetische Beziehung zwischen Autor und Referenztext sowie werkästhetische Beziehung zwischen Phäno- und Referenztext programmieren wiederum - und eben darin liegt die appellative Funktion dieser Signalzeichen - ein spezifisches Leseverhalten. Dieses den Referenzsignalen implizite Rezeptionsprogramm informiert den Leser sowohl über das gegenständliche ,Was' der für das Verstehen des literarischen Texts notwendigen weiteren Textlektüren als auch über das dialogische ,Wie'. Denn die „Kommunikationssituation, in der der Text als Replik entsteht“‘316 und die in die Replik eingeht, evoziert ihrerseits ein Kommunikationsverhalten, das nach

314 Die Unterscheidung zwischen dem ,Ausloten` bzw. Eröffnen der Differenzen einerseits und den darauf sich gründenden Semantisierungsprozessen andererseits wird hier aus heuristischen Gründen getroffen. Selbstverständlich gehört die Ausdifferenzierung dieser Differenzen zum Vorgang der Semantisierung. Gleichwohl können diese Prozesse der Bedeutungs- und Sinnkonstitutierung erst dann einsetzen, nachdem das Vorhandensein einer Differenz bemerkt wurde, der Leser also in welcher Weise auch immer in seiner Lektüre gestört oder irritiert wird.

315 Der Text muss also die Neugier des Lesers provozieren, die im Falle der poetica scientiae als theoretische bzw. szientifische Neugier beschrieben werden kann; ihr wohnt ein energetischer Aspekt inne, der zur Spurensuche anleitet und zur Lektüre anderer Textquellen motiviert. Der Terminus ,theoretische Neugierde“ ist entlehnt von Hans Blumenberg: Der Prozeß der theoretischen Neugierde, Frankfurt/M. 1979.

316 Lachmann: Bachtins Dialogizität, S. 509. 
der Wiederholung der dem Text vorausliegenden Kommunikationssituation verlangt, in deren Verlauf die in den Text eingesenkten heterogenen Elemente in ihrer ursprünglichen textuellen Ordnung wiederhergestellt werden, „ohne dabei die Sinnkomplexion abzubauen, die in der (ludistischen) Heterogenisierung der Elemente erzeugt werden konnte“. 317

Die Funktionen, die Referenz- und Fiktionssignale innerhalb des Leseprozesses übernehmen, sind fundamental verschiedene (auch dann, wenn beide in einem einzigen linguistischen Zeichen zusammenfallen). Die Fiktionssignale bedeuten dem Leser, dass die zur Sprache gebrachte Welt „nicht eine gegebene Welt ist, sondern daß sie so verstanden werden soll, als ob sie eine gegebene Welt sei“. ${ }^{318}$ Diese Vorstellung im Zeichen des Als-ob impliziert eine doppelte Referenz, insofern sie auf die in ihr überschrittene wirkliche Welt verweist (der Verweis eröffnet überhaupt erst die Vorstellbarkeit) und zugleich bedeutet, dass die vorgestellte Welt nicht Selbstzweck ist - ,es handelt sich doch nicht darum, ohne jeden Zweck etwas Unwirkliches als wirklich anzunehmen“ -, ${ }^{319}$ sondern sich in ihr die Dimension eines pragmatischen Gebrauchs abschattet. ${ }^{320}$ Relevant für den aktuellen Zusammenhang ist, dass das durch die Fiktionssignale angezeigte Fiktive eine Irrealisierung sowohl der realen Welt als auch des Lesers erwirkt, d.h. empirische Lebenswelt und empirisches Lesersubjekt depotenzieren sich zu einem Analogon, „damit durch dieses hindurch einer irrealen Welt die Möglichkeit für ihr reales Erscheinen gewinnen kann“.321 In der Folge dieses Realwerdens des Fiktiven wird der Als-ob-Charakter (wenigstens vorübergehend) suspendiert bzw. vergessen: Das Realwerden des Fiktiven verwandelt das ,Es ist, als ob“ in ein ,Es ist‘.

317 Lachmann: Ebenen des Intertextualitätsbegriffs, S. 137.

318 Iser: Akte des Fingierens, S.139.

319 So bereits Hans Vaihinger: Die Philosophie des Als ob, Leipzig ${ }^{8} 1922$, S. 589, hier zitiert nach Iser: Akte des Fingierens, S. 141.

320 Mit der Fiktion des Als-ob erfolge, so Iser, eine doppelte Überschreitung, nämlich einerseits die Überschreitung der wirklichen Welt in den Akten des Fingierens, und andererseits eine Überschreitung der fiktionalen Welt selbst. Die dargestellte Welt des Textes lasse sich nicht mit der wirklichen Welt mimetisch verrechnen; vielmehr sei sie ein Sachverhalt, der gerade durch seine Als-ob-Kennzeichnung weder seine Bestimmung noch seine Wahrheit in sich selber tragen könne, sondern diese immer nur in Beziehung zu etwas anderem suchen und finden müsse (vgl. Iser: Akte des Fingierens, S. 142-144). - Nach Iser zielt diese durch das Alsob hergestellte Gebrauchsfunktion letztlich auf eine ästhetische Erfahrung: Diese könne sowohl Reaktion auf die Textwelt als auch Reaktion auf die empirische Welt sein, ,die durch das Analogon der Textwelt hindurch aus einer Perspektive visiert wird, die nicht eine solche der gegebenen Lebenswelt ist“" (ebd., S. 145).

321 Ebd., S. 146. 
Die szientifischen Referenzsignale hingegen depotenzieren das Lesersubjekt gerade nicht zu einem Analogon zum Zwecke des Realwerdens des Fiktiven, sondern erinnern es gleichsam an seinen empirischen Status: In ihrer Funktion als Appellative distanzieren sie den Leser von der fiktionalen Welt, stören und unterbrechen den Prozess der Realisierung der fiktionalen Welt und holen die Differenz zwischen Realem und Fiktivem ins Bewusstsein zurück. Die szientifischen Referenzsignale, sofern sie als solche identifiziert werden, provozieren sodann eine Lektüre, die kompensatorisch die jeweiligen ursprünglichen textuellen Ordnungen wiederherstellt; mit anderen Worten: die durch sie angezeigte metonymische Relation verlangt die Wiederherstellung jenes (Gesamt-)Kontextes, dessen Verkürzung die Metonymie ist und dessen sie zu ihrem Funktionieren bedarf. ${ }^{322}$

Je mehr ein fiktionaler Text die Depotenzierung und Irrealisierung von empirischer Welt und empirischem Subjekt ermöglicht, je vollkommener also die Realisierung der irreal-fiktionalen Welt gelingt, desto stärker erfährt sich der Leser in die fiktionale Welt hineingenommen, desto stärker ist deren Als-obCharakter außer Kraft gesetzt. Je prominenter (und vielleicht auch enigmatischer) hingegen die Referenzsignale gesetzt sind, desto mehr wird sich der Leser angehalten sehen, die fiktionale Welt vorübergehend $\mathrm{zu}$ verlassen und sich die Kontexte in der Lektüre jener Texte anzueignen, auf welche die metonymische Relation verweist bzw. solcher Texte, die den wissenschaftlichen Zusammenhang der metonymischen Verkürzung herstellen helfen. ${ }^{323}$

Die (nachträgliche) Lektüre der Prätexte wird dabei immer bis zu einem gewissen Grad von der (zuvor erfolgten) Lektüre des aktuellen Textes beeinflusst sein: Lektüreumfang, Kenntnisaneignung oder -vertiefung, Ausmaß der Kontextualisierung der in den aktuellen Text eingelagerten Wissenselemente etc. ge-

322 Diese Wiederherstellung des Gesamtkontexts kann auf unterschiedliche Weise erfolgen und hängt in jedem Fall davon ab, bis zu welchem Grad der Leser über diesen Kontext bereits verfügt und in welchem Maße er am Verstehen jener metonymischen Relation interessiert ist.

323 Man könnte in diesem Zusammenhang von bestimmten Referenztexten einerseits, von adjutanten Referenztexten andererseits sprechen. - Ein Referenzsignal kann - abhängig von dem Grad, in dem das Realwerden des Fiktiven geschieht - die Funktion eines Fiktionssignals übernehmen, indem es die Differenz von Fiktion und Wirklichkeit wieder ins Spiel bringt und den fiktiven Kontext, in dem es als nicht-fiktionales Zeichen steht, erinnert. - Die (Wieder)Herstellung des Referenztextes entspricht der Herstellung einer Refigurationsdisposition, d.h. sie ist innerhalb des Lektüreprozesses lediglich ein erstes Stadium, in dem sich der Leser in die Lage versetzt, die Refiguration zu leisten. Zum rezeptionsästhetischen Begriff der Refiguration, von Ricœur als mimêsis III gefasst, vgl. Paul Ricœur: Zeit und Erzählung, Bd. I: Zeit und historische Erzählung, München 1988, S. 113 f. 
schieht nicht unabhängig vom aktuellen Text, sondern wird von diesem, konkreter: von dem durch ihn aufgeworfenen Fragen, von dem in ihm gegebenen Antworten und Deutungen etc. überlagert und gesteuert. Auf diese Weise wirkt der aktuelle Text auf den durch ihn hindurchgegangenen, wie auch immer transformierten und reorganisierten Ursprungstext zurück. Wie der aktuelle Text in der Kommunikation mit einem fremden Text als Replik entsteht, wobei die Kommunikationssituation in die Replik selbst eingeht - „der Text wird so zum Bestandteil der ,realen' Situation, der nur in der Synthese mit ihr ein Zeichen bildet“ - ${ }^{324}$ so entsteht umgekehrt auch der Referenztext in der Kommunikation mit dem nun durch den Leser hindurchgegangenen und wie auch immer verstandenen aktuellen Text als Replik, genauer: als Replik auf die (vorläufige) Leserreplik auf den aktuellen, seinerseits replikativ auf den Ursprungstext bezogenen aktuellen Text. In diesem Stadium der Rezeption kehrt sich das Verhältnis zwischen den Texten um: Der ,eigentliche‘, frühere Prätext erscheint in der Lektüre als Folgetext des ,eigentlichen', späteren Folgetexts.

Diese durch die appellative Funktion der Referenzsignale ausgelöste und im Horizont des aktuellen Textes erfolgende Lektüre der Prätexte ist Teil der poetologischen Intentionalität der poetica scientiae, mehr noch: Bedingung der Möglichkeit der Realisierung ihrer ästhetischen und erkenntnisstiftenden Funktionen. Die Lektüre der intertextuell markierten Prätexte ist integratives und poetologisch kalkuliertes Moment der Lektüre des aktuellen Textes. Dessen Sinn- und Bedeutungspotential weist stets über die eigenen Textgrenzen hinaus und zurück auf den fremden Text, der erst durch die im Deutungshorizont des aktuellen Texts sich ereignende Lektüre eine neue und andere, in ihm zwar potentiell angelegte, aber bis dahin nicht aktualisierte Perspektive, Konturiertheit, Werthaltigkeit, Prägnanz, Kohärenz oder auch Diskontinuität und Offenheit gewinnt. Erst durch diese Einflussnahme, die der aktuelle Text auf die Lektüre und Interpretation des fremden, szientifischen Textes - auf die in ihm thematisierten Sachverhalte, auf seine sprachlich-rhetorische Gestalt, auf die ihm möglicherweise eigenen Brüche und Leerstellen etc. - ausübt, vermag der aktuelle Text seine spezifisch intertextuellen Funktionen zu erfüllen; erst durch diese vom Leser zu aktualisierende Rück-Wirkung auf den fremden Bezugstext vermag der aktuelle seine Einstellung und Haltung - sei es eine kritischprüfende, kommentierende, interpretierende, affirmative, negierende - jenem gegenüber $\mathrm{zu}$ evozieren und damit sein metatextuelles Potential $\mathrm{zu}$ verwirklichen. In dem Maße, in dem der fremde Text im Deutungsrahmen des aktuellen einer (Wieder-)Lektüre unterzogen wird, er sich auf diese Weise für eine andere

324 Lachmann: Bachtins Dialogizität, S. 509. 
Sprach-, Gestalt- und Sinnwahrnehmung öffnet, seine Monologizität durch erinnerte (wissenschafts-)historische oder ideologische Kontexte, durch in ihm präsente Intertexte oder Begriffsgeschichten zur Vielstimmigkeit aufgesprengt wird, in dem Maße werden sich auch die intertextuellen und intratextuellen Differenzen des aktuellen Textes zeigen, seine Polyphonie, Polyvalenz und damit seine Verschiedenverstehbarkeit potenziert.

Lektürepraktisch geschieht dies jedoch erst in einem weiteren Schritt, der zumeist in der Relektüre des fiktionalen Textes - nun freilich auf der Grundlage des angeeigneten szientifischen Kontextes und damit der vom Leser erworbenen Replikkompetenz - besteht. Erst jetzt wird die Rückkehr in die fiktionale Welt, werden neue, andere, vielfältigere Semantisierungs-, Verstehens- und Reflexionsprozesse erfolgen. Diese Lektüre lässt sich als eine Art synoptische Lektüre begreifen: Sie erfolgt synchron mit der erinnerten Lektüre des/der fremden Texte(s), und es ist eben dieser erinnernd mitgelesene Subtext, der die lineare Lektüre des aktuellen Textes nachhaltig stört und unterbricht, seine Entklammerung aus dem Als-ob und damit eine störungsfreie, identifikatorische Lektüre verhindert. Einmal stimmhaft geworden, kann der Referenztext nicht mehr in die Stummheit zurückfallen; ${ }^{325}$ er wird sich im Prozess der Lektüre immer wieder einmischen, sich Geltung verschaffen, mitreden, mitdeuten und sich - abhängig vom quantitativen und qualitativen Ausmaß der Referenzsignale sowie von der quantitativen und qualitativen Präsenz, die der abwesende, fremde Text im Leser innehat - mal als Hintergrundgeräusch, mal als gleichberechtigte Mit-Stimme, mal als über die Stimmen des fiktionalen Textes dominierende Stimme ,zu Gehör bringen‘. Entsprechend variabel gestaltet sich auch das Verhältnis der Texte zueinander: Der fiktionale Text kann seine Dominanz über den szientifischen behalten, aber auch temporär einbüßen, beide Texte können auch gleichberechtigt nebeneinander existieren. Wie die Lektüre des fremden Textes zum poetologischen Kalkül des aktuellen Textes gehört, so auch das Risiko, vom fremden Text verdrängt zu werden, d.h. das Risiko, dass sich das Leserinteresse völlig auf den fremden Text verlagert. - Der Leser ist dabei stets die vermittelnde Instanz zwischen Prä- und Folgetext: Er ist diejenige Instanz, die den lediglich metonymisch präsenten Prätext vertritt und in der Entfaltung seiner Kontexte den Dialog zwischen Prä- und Folgetext inszeniert.

325 Diese Aussage hat nur insoweit Gültigkeit, als der Referenztext tatsächlich im Leser präsent ist. Eine genaue Phänomenologie des Lektüreverhaltens müsste den zeitlichen Abstand, der zwischen den Lektüren liegt, berücksichtigen, müsste ferner dem Umstand Rechnung tragen, dass kulturelles und szientifisches Wissen (temporär) auch völlig vergessen werden kann. 
Die intertextuelle Lektüre ist damit stets eine dialektische, ein Hin- und Hergehen zwischen den Texten, ein Ereignis, das gleichsam zwischen den Texten bzw. auf der Grenze der Texte stattfindet. Die für die Gattung poetica scientiae charakteristische Kontiguitätspoetik erhält ihr rezeptionsästhetisches Pendant in einer Kontiguitätslektüre, die hier wie dort in eine Kontiguitätshermeneutik ausmündet: Ebenso wie Sinn im Text durch die Berührung und Kreuzung zweier Horizonte - also: dialogisch - entsteht und sich als Antwort auf Zeichen zeichenhaft materialisiert, ist das Sinnverstehen ein Akt der Berührung und Kreuzung, durch den der aktuelle Text und die in ihm punktuell eingefrorenen Sinnidentitäten ihre Dialogizität - und damit ihre Kontiguitäts- und Differenzqualität - wiedererlangen, woraus möglicherweise neue differenz- und dialogaufhebende Antworten entstehen. Sinnkonstitution und Sinnverstehen meint hier programmatisch: Partizipation am Fremden und Anderen. Diese Teilhabe ist keine beliebige und kontingente, sondern eine intendierte und entsprechend ausgewiesene.

Der (selbstreflexive) Rückverweis des literarischen Textes auf seine Genese in der Kommunikation mit einem fremden Text, ist zugleich Vorverweis auf einen Lektüre- und Verstehensweg - ganz buchstäblich also auf eine Rezeptionsmethode -, dessen ,Abschreiten“ einen Lesertypus von der Art ,erzeugt", wie ihn der Text zu seinem Verständnis voraussetzt. Der lector doctus, dessen die Gattung poetica scientiae zu ihrer Realisierung bedarf, kann in der Regel nicht als selbstverständlich gegeben vorausgesetzt werden, sondern wird mit Hilfe der im Text programmierten sachlichen und methodischen Referenzen allererst generiert. 
